ESAIM: M2AN

Vol. 41, No 3,2007 , pp. 513-542

DOI: $10.1051 / \mathrm{m} 2 \mathrm{an}: 2007033$
ESAIM: Mathematical Modelling and Numerical Analysis

www.edpsciences.org/m2an

\title{
COMPARAISON ENTRE MODÈLES D'ONDES DE SURFACE EN DIMENSION 2
}

\author{
YOUCEF MAMMERI ${ }^{1}$
}

\begin{abstract}
Résumé. Partant du principe de conservation de la masse et du principe fondamental de la dynamique, on retrouve l'équation d'Euler nous permettant de décrire les modèles asymptotiques de propagation d'ondes dans des eaux peu profondes en dimension 1. Pour décrire la propagation des ondes en dimension 2, Kadomtsev et Petviashvili [Sov. Phys. Dokady 15 (1970) 539] utilisent une perturbation linéaire de l'équation de KdV. Mais cela ne précise pas si les équations ainsi obtenues dérivent de l'équation d'Euler, c'est ce que montrent Ablowitz et Segur dans l'article [J. Fluid Mech. 92 (1979) 691-715]. On insistera, de la même manière, sur le fait que les équations de KP-BBM peuvent être aussi obtenues à partir de l'équation d'Euler, et dans quelle mesure elles décrivent le modèle physique. Dans un second temps, on reprend la méthode introduite dans l'article de Bona et al. [Lect. Appl. Math. 20 (1983) 235-267] dans lequel ils comparent les solutions d'ondes longues en dimension 1, à savoir les solutions des équations KdV et BBM, pour montrer ici que les solutions des équations KP-II et KP-BBM-II sont proches sur un intervalle de temps inversement proportionnel à l'amplitude des ondes. Du point de vue de la modélisation, il sera clair, d'après la première partie, que seul le modèle décrit par KP-BBM-II est bien posé, et comme du point de vue physique, KP-II et KP-BBM-II décrivent les ondes longues de faible amplitude lorsque la tension de surface est négligeable, il est intéressant de les comparer. De plus, on verra que la méthode utilisée ici reste valable pour les problèmes périodiques.
\end{abstract}

\begin{abstract}
On the basis of the principle of conservation of mass and fundamental principle of dynamics, we find the Euler equation enabling us to describe the asymptotic models of waves propagation in shallow water in dimension 1 . To describe the waves propagation in dimension 2, a linear perturbation of the KdV equation is used by Kadomtsev and Petviashvili [Sov. Phys. Dokady 15 (1970) 539]. But that does not specify if the equations thus obtained derive from the Euler equation, that is shown by Ablowitz and Segur in [J. Fluid Mech. 92 (1979) 691-715]. We will insist, in same manner, on the fact that the equations of KP-BBM can be also obtained starting from the Euler equation, and up to what point they describe the physical model. In a second time, we take again the method introduced in the article of Bona et al. [Lect. Appl. Math. 20 (1983) 235-267] in which the solutions of long water waves in dimension 1, namely the solutions of $\mathrm{KdV}$ and $\mathrm{BBM}$, are compared, to show here that the solutions of KP-II and KP-BBM-II are close for a time scale inversely proportional to the waves amplitude. From the point of view of modelling, it will be clear according to the first part, that only the model described by KP-BBM-II is well posed, and since from the physical point of view, KP-II and KP-BBM-II describe the small amplitude long waves when the surface tension is neglected, it is interesting to compare them. Moreover, we will see that the method used here remains valid for the periodic problems.
\end{abstract}

Classification Mathématique. 35B05, 35B10, 35Q53, 65M99, 76B15.

Reçu le 1er août 2006. Revisé le 23 janvier 2007.

Mots Clés. KP, KP-BBM equations, models derivation, comparison, relaxation method.

1 Laboratoire Paul Painlevé, Université des Sciences et Technologies Lille 1, France. youcef.mammeri@math.univ-lille1.fr

(c) EDP Sciences, SMAI 2007 


\section{INTRODUCTION}

Dans ce papier, nous étudions certains modèles asymptotiques des ondes hydrodynamiques. On s'intéresse plus particulièrement aux modèles d'ondes longues de faible amplitude se propageant à la surface de l'eau en dimensions 1 et 2 . L'écoulement du flux irrotationnel d'un fluide parfait incompressible homogène est décrit par l'équation d'Euler

$$
\frac{\partial u}{\partial t}+\nabla\left(\frac{u^{2}}{2}\right)=-\nabla\left(\frac{P}{\rho_{0}}\right)-\nabla g z
$$

où $u \in \mathbb{R}^{3}$ désigne le champ de vitesse. À partir de cette équation, on obtient les équations décrivant la surface libre $\eta$ de ces ondes en dimension 1, à savoir les équations de Korteweg-de Vries (KdV) [15]

$$
\eta_{t}+\eta_{x}+\frac{3}{2} \alpha \eta \eta_{x}+\frac{1}{2}\left(\frac{1}{3}-\sigma\right) \beta \eta_{x x x}=0,
$$

et de Benjamin-Bona-Mahony (BBM) [2], pour $\sigma<1 / 3$,

$$
\eta_{x}+\eta_{t}+\frac{3}{2} \alpha \eta \eta_{x}-\frac{1}{2}\left(\frac{1}{3}-\sigma\right) \beta \eta_{x x t}=0,
$$

où $\sigma>0$ désigne le nombre de Bond lié à la tension de surface, $\alpha$ est le quotient entre l'amplitude des ondes et la profondeur de l'eau, et $\beta$ est le carré du quotient entre la profondeur et la longueur d'onde. En dimension 2 , les modèles asymptotiques sont donnés par les équations de Kadomstev-Petviashvili [13]

$$
\left(\eta_{t}+\eta_{x}+\frac{3}{2} \alpha \eta \eta_{x}+\frac{1}{2}\left(\frac{1}{3}-\sigma\right) \beta \eta_{x x x}\right)_{x}+\frac{\gamma}{2} \eta_{y y}=0
$$

appelées KP-I si $\sigma>1 / 3$, KP-II si $\sigma<1 / 3$, où $\gamma$ désigne ici le carré du quotient entre les longueurs d'onde dans les deux directions du plan.

De la même manière que l'on passe de KdV à BBM, on peut se demander quels sont les modèles correspondant aux équations KP-I et KP-II. Dans un premier temps, on vérifiera que seul le modèle décrit par l'équation KPBBM-II

$$
\left(\eta_{t}+\eta_{x}+\frac{3}{2} \alpha \eta \eta_{x}-\frac{1}{2}\left(\frac{1}{3}-\sigma\right) \beta \eta_{x x t}\right)_{x}+\frac{\gamma}{2} \eta_{y y}=0
$$

pour $\sigma<1 / 3$, est linéairement bien posé dans $L^{2}\left(\mathbb{R}^{2}\right)$, et donc est le seul modèle raisonnable. De même, BBM est un modèle raisonnable uniquement pour $\sigma<1 / 3$.

Une fois ce travail de modélisation effectué, on pourra, dans un second temps, comparer les solutions des équations de KP-II et KP-BBM-II. On supposera alors que $\alpha, \beta$ et $\gamma$ sont égaux.

Avant d'énoncer notre résultat, rappelons que Bona et al. [6] ont montré en dimension 1 :

Théorème 0.1. Soient $m \geq 1$ et $f \in H^{m+5}(\mathbb{R})$. Soit $\alpha>0$ et soient $\eta$ et $\zeta$ respectivement l'unique solution de $\mathrm{KdV}$ et de $\mathrm{BBM}$ dans $\mathcal{C}\left(\left[0, \infty\left[; H^{m+5}(\mathbb{R})\right)\right.\right.$, sous la condition initiale $\left.\eta\right|_{t=0}=\left.\zeta\right|_{t=0}=f$. Alors, il existe $\alpha_{0}>0$, et pour $0 \leq i \leq m-1$, une constante d'ordre $1 M_{i}>0$, telle que si $0<\alpha \leq \alpha_{0}$, et pour $0 \leq t \leq \alpha^{-1}$, on ait

$$
\left\|\partial_{x}^{i} \eta(., t)-\partial_{x}^{i} \zeta(., t)\right\|_{L^{\infty}(\mathbb{R})} \leq M_{i} \alpha^{2} t
$$

Pour les modèles de dimension 2 sans tension de surface, on montrera un résultat similaire. Si on note $X^{m}\left(\mathbb{R}^{2}\right)$ l'ensemble des fonctions $f$ de $H^{m}\left(\mathbb{R}^{2}\right)$ telles que $\partial_{x}^{-1} f$ soit dans $H^{m}\left(\mathbb{R}^{2}\right)$, on peut énoncer :

Théorème 0.2. Soient $m \geq 2$ et $f \in X^{m+5}\left(\mathbb{R}^{2}\right)$. Soit $\alpha>0$ et soient $\eta$ et $\zeta$ respectivement l'unique solution de KP-II et de KP-BBM-II dans $\mathcal{C}\left([-T, T] ; X^{m+5}\left(\mathbb{R}^{2}\right)\right)$, avec $T:=\frac{\alpha^{-1}}{C_{m+5}|f|_{m+5}}$, sous la condition initiale 
$\left.\eta\right|_{t=0}=\left.\zeta\right|_{t=0}=f$. Alors, il existe $\alpha_{0}>0$, et pour tous entiers naturels $0 \leq i+j \leq m-2$, une constante $N_{i, j}>0$, dépendant uniquement de la norme de $f$ dans $X^{m+5}\left(\mathbb{R}^{2}\right)$, telle que si $0<\alpha \leq \alpha_{0}$, et pour $|t| \leq T$, on ait

$$
\left\|\partial_{x}^{i} \partial_{y}^{j} \eta(., ., t)-\partial_{x}^{i} \partial_{y}^{j} \zeta(., ., t)\right\|_{L^{\infty}\left(\mathbb{R}^{2}\right)} \leq N_{i, j} \alpha^{3 / 2}|t|^{1 / 2}
$$

La preuve consistera à étudier les solutions des équations

$$
\begin{aligned}
u_{t}+u u_{x}+u_{x x x}+\partial_{x}^{-1} u_{y y} & =0 \\
v_{t}+v v_{x}+v_{x x x}-\alpha v_{x x t}+\partial_{x}^{-1} v_{y y} & =0 .
\end{aligned}
$$

La chose importante est de remarquer que, lorsque l'on prend $\alpha=\beta=\gamma$, le passage de KP-II à (0.1) et de KP-BBM-II à (0.2) se fait uniquement à l'aide du changement de variables

$$
u(x, y, t)=\eta\left(x+\alpha^{-1} t, y, \alpha^{-1} t\right), v(x, y, t)=\zeta\left(x+\alpha^{-1} t, y, \alpha^{-1} t\right) .
$$

Clairement, ce changement de variables nous permettra de généraliser nos résultats à des problèmes périodiques dans les deux directions d'espace.

On pourra alors s'intéresser à cette comparaison de façon numérique en se restreignant à des domaines de type $[-\pi, \pi] \times[-\pi, \pi]$, puis en prolongeant par périodicité. On se demandera alors si l'intervalle du temps de comparaison pourrait être plus grand. On observera, effectivement, que les inégalités de comparaison restent vraies au-delà du temps donné par le théorème précédent, et que le comportement du problème non linéaire est semblable à celui du problème linéaire lors des premiers instants d'observation.

\section{DÉRIVATION DE KP-BBM}

On s'appuie ici essentiellement sur les livres [17,20]. Jusqu'à la Section 2, les résultats seront formels.

On se place dans un référentiel galiléen de $\mathbb{R}^{3}$, c'est-à-dire qu'on se munit d'un repère orthonormé $(O x, O y, O z)$ avec $O z$ vertical dirigé vers le haut et d'une chronologie, pour nous permettre d'étudier les aspects cinématiques de l'écoulement du fluide. On considère un flux irrotationnel d'un fluide parfait incompressible homogène remplissant le volume défini par $-h<z<\eta(x, y, t)$ et $(x, y) \in \mathbb{R}^{2}$ tel que la surface libre du fluide soit décrite par l'équation $z=\eta(x, y, t)$. On note $\rho_{0}$ la constante représentant la masse volumique du système et $T$ la tension de surface.

Il existe une fonction potentielle de vitesse $\varphi$ vérifiant

$$
\begin{aligned}
& \varphi_{x x}+\varphi_{y y}+\varphi_{z z}=0 \text { si }-h<z<\eta(x, y, t), \\
& \varphi_{t}+\frac{1}{2}\left(\varphi_{x}^{2}+\varphi_{y}^{2}+\varphi_{z}^{2}\right)+g \eta \\
& -\frac{T}{\rho_{0}} \frac{\eta_{x x}\left(1+\eta_{y}^{2}\right)+\eta_{y y}\left(1+\eta_{x}^{2}\right)-2 \eta_{x y} \eta_{x} \eta_{y}}{\left(1+\eta_{x}^{2}+\eta_{y}^{2}\right)^{3 / 2}}=0 \quad \text { si } z=\eta(x, y, t) \text {, } \\
& \eta_{t}+\varphi_{x} \eta_{x}+\varphi_{y} \eta_{y}-\varphi_{z}=0 \text { si } z=\eta(x, y, t), \\
& \varphi_{z}=0 \text { si } z=-h \text {. }
\end{aligned}
$$

L'existence de cette fonction $\varphi$ est due au fait que le flux est irrotationnel ([19], Chap. 1 - Sect. 13), à cela s'ajoute la considération d'un fluide homogène ce qui donne l'équation (1.1) ([17], Chap. 8 - Sect. 1). L'équation (1.2) est l'équation d'Euler incompressible, elle découle directement du principe fondamental de la dynamique ([17], Chap. 7 - Sect. 1). L'équation (1.3) est une condition cinématique, elle traduit le fait que la vitesse d'écoulement du fluide est tangente à la surface. Enfin l'équation (1.4) impose une condition de non pénétration sur la surface intérieure ([17], Chap. 18 - Sect. 1).

On suppose que l'équation de la surface vérifie $\eta=O(a)$, où $a$ est l'amplitude des ondes. On suppose de plus que l'amplitude des ondes a est négligeable par rapport à la profondeur $h$ et que cette profondeur $h$ est 
elle-même négligeable par rapport à $k$ la longueur d'onde dans la direction $x$, c'est-à-dire que l'on s'intéresse aux ondes de faible amplitude et de grande longueur. Mais on suppose aussi que $k$, la longueur d'onde dans la direction $x$, est négligeable par rapport à $l$, la longueur d'onde dans la direction $y$, c'est-à-dire que l'écoulement se fait principalement dans la direction $x$.

On cherche à adimensionner les équations précédentes. Pour cela, on suppose que, pour $c=\sqrt{g h}$, la vitesse caractéristique des ondes, les fonctions $\varphi$ et $\eta$ s'écrivent

$$
\begin{aligned}
\varphi(x, y, z, t) & =\frac{g k a}{c} \widetilde{\varphi}\left(\frac{x}{k}, \frac{y}{l}, \frac{z}{h}+1, \frac{c}{k} t\right), \\
\eta(x, y, t) & =a \widetilde{\eta}\left(\frac{x}{k}, \frac{y}{l}, \frac{c}{k} t\right) .
\end{aligned}
$$

Ce changement de fonctions est important, il nous permet de passer de fonctions qui peuvent être très oscillantes, donc difficiles à manipuler, ici $\varphi$ et $\eta$, à des fonctions avec beaucoup moins d'oscillations, autrement dit de grande longueur d'ondes, ici $\widetilde{\varphi}$ et $\widetilde{\eta}$. On pose

$$
\begin{aligned}
& \widetilde{x}=\frac{x}{k}, \quad \widetilde{y}=\frac{y}{l}, \quad \widetilde{z}=\frac{z}{h}+1, \quad \widetilde{t}=\frac{c}{k} t, \\
& \alpha=\frac{a}{h}, \quad \beta=\frac{h^{2}}{k^{2}}, \quad \gamma=\frac{k^{2}}{l^{2}}, \quad T=h^{2} \rho_{0} g \sigma,
\end{aligned}
$$

où $\sigma>0$ désigne toujours le nombre de Bond. Par abus, on supprimera les tildes dans les équations obtenues par ces changements. L'équation de la surface devient $z=1+\alpha \eta$, et les équations (1.1), (1.2), (1.3) et (1.4)

$$
\begin{aligned}
& \beta \varphi_{x x}+\beta \gamma \varphi_{y y}+\varphi_{z z}=0 \text { si } 0<z<1+\alpha \eta, \\
& \varphi_{t}+\frac{1}{2} \alpha \varphi_{x}^{2}+\frac{1}{2} \alpha \gamma \varphi_{y}^{2}+\frac{1}{2} \frac{\alpha}{\beta} \varphi_{z}^{2}+\eta \\
& -\beta \sigma \frac{\eta_{x x}\left(1+\alpha^{2} \beta \gamma \eta_{y}^{2}\right)+\gamma \eta_{y y}\left(1+\alpha^{2} \beta \eta_{x}^{2}\right)-2 \alpha^{2} \beta \gamma \eta_{x y} \eta_{x} \eta_{y}}{\left(1+\alpha^{2} \beta \eta_{x}^{2}+\alpha^{2} \beta \gamma \eta_{y}^{2}\right)^{3 / 2}}=0 \quad \text { si } z=1+\alpha \eta, \\
& \eta_{t}+\alpha \varphi_{x} \eta_{x}+\alpha \gamma \varphi_{y} \eta_{y}-\frac{1}{\beta} \varphi_{z}=0 \quad \text { si } z=1+\alpha \eta, \\
& \varphi_{z}=0 \text { si } z=0 \text {. }
\end{aligned}
$$

Vérifions que l'on retrouve bien les équations précédentes.

(i) L'équation de Laplace (1.1) donne

$$
\frac{1}{k^{2}} \widetilde{\varphi}_{\widetilde{x} \widetilde{x}}+\frac{1}{l^{2}} \widetilde{\varphi}_{\tilde{y} \widetilde{y}}+\frac{1}{h^{2}} \widetilde{\varphi}_{\widetilde{z} \widetilde{z}}=0
$$

Multiplier par $h^{2}$ donne (1.5).

(ii) L'équation (1.2) donne

$$
\begin{aligned}
& g a \widetilde{\eta}+ \frac{g k a}{c} \frac{c}{k} \widetilde{\varphi}_{\widetilde{t}}+\frac{1}{2}\left(\left(\frac{g k a}{c}\right)^{2} \frac{1}{k^{2}} \widetilde{\varphi}_{\widetilde{x}}^{2}+\left(\frac{g k a}{c}\right)^{2} \frac{1}{l^{2}} \widetilde{\varphi}_{\widetilde{y}}^{2}+\left(\frac{g k a}{c}\right)^{2} \frac{1}{h^{2}} \widetilde{\varphi}_{\widetilde{z}}^{2}\right) \\
&-g h^{2} \sigma \frac{\frac{a}{k^{2}} \widetilde{\eta}_{\widetilde{x} \widetilde{x}}\left(1+\frac{a^{2}}{l^{2}} \widetilde{\eta}_{\widetilde{y}}^{2}\right)+\frac{a}{l^{2}} \widetilde{\eta}_{\widetilde{y} \widetilde{y}}\left(1+\frac{a^{2}}{k^{2}} \widetilde{\eta}_{\widetilde{x}}^{2}\right)-2 \frac{a^{3}}{k^{2} l^{2}} \widetilde{\eta}_{\widetilde{x} \widetilde{y}} \widetilde{\eta}_{\widetilde{x}} \widetilde{\eta}_{\widetilde{y}}}{\left(1+\frac{a^{2}}{k^{2}} \widetilde{\eta}_{\widetilde{x}}^{2}+\frac{a^{2}}{l^{2}} \widetilde{\eta}_{\widetilde{y}}^{2}\right)^{3 / 2}}=0 .
\end{aligned}
$$


En divisant par $g a$, on trouve

$$
\begin{gathered}
\widetilde{\eta}+\widetilde{\varphi}_{\widetilde{t}}+\frac{1}{2}\left(\frac{g a}{c^{2}} \widetilde{\varphi}_{\widetilde{x}}^{2}+\frac{g a}{c^{2}} \frac{k^{2}}{l^{2}} \widetilde{\varphi}_{\widetilde{y}}^{2}+\frac{g a}{c^{2}} \frac{k^{2}}{h^{2}} \widetilde{\varphi}_{\widetilde{z}}^{2}\right) \\
-\frac{h^{2}}{k^{2}} \sigma \frac{\widetilde{\eta}_{\widetilde{x} \widetilde{x}}\left(1+\frac{a^{2}}{h^{2}} \frac{h^{2}}{k^{2}} \frac{k^{2}}{l^{2}} \widetilde{\eta}_{\widetilde{y}}^{2}\right)+\frac{k^{2}}{l^{2}} \widetilde{\eta}_{\widetilde{y} \widetilde{y}}\left(1+\frac{a^{2}}{h^{2}} \frac{h^{2}}{k^{2}} \widetilde{\eta}_{\widetilde{x}}^{2}\right)-2 \frac{a^{2}}{h^{2}} \frac{h^{2}}{k^{2}} \frac{k^{2}}{l^{2}} \widetilde{\eta}_{\widetilde{x} \widetilde{y}} \widetilde{\eta}_{\widetilde{x}} \widetilde{\eta}_{\widetilde{y}}}{\left(1+\frac{a^{2}}{h^{2}} \frac{h^{2}}{k^{2}} \widetilde{\eta}_{\widetilde{x}}^{2}+\frac{a^{2}}{h^{2}} \frac{h^{2}}{k^{2}} \frac{k^{2}}{l^{2}} \widetilde{\eta}_{\widetilde{y}}^{2}\right)^{3 / 2}}=0 .
\end{gathered}
$$

qui est l'équation (1.6).

(iii) L'équation (1.3) donne

$$
\frac{a c}{k} \widetilde{\eta}_{\tilde{t}}+\frac{g a^{2}}{k c} \widetilde{\varphi}_{\widetilde{x}} \widetilde{\eta}_{\widetilde{x}}+\frac{g a^{2}}{c} \frac{k}{l^{2}} \widetilde{\varphi}_{\widetilde{y}} \widetilde{\eta}_{\widetilde{y}}-\frac{g k a}{c h} \widetilde{\varphi}_{\widetilde{z}}=0
$$

En divisant par $\frac{a c}{k}$, on trouve

$$
\widetilde{\eta}_{\tilde{t}}+\frac{g a}{c^{2}} \widetilde{\varphi}_{\widetilde{x}} \widetilde{\eta}_{\widetilde{x}}+\frac{g a}{c^{2}} \frac{k^{2}}{l^{2}} \widetilde{\varphi}_{\widetilde{y}} \widetilde{\eta}_{\widetilde{y}}-\frac{g}{c^{2}} \frac{k^{2}}{h} \widetilde{\varphi}_{\widetilde{z}}=0
$$

qui est l'équation (1.7).

On veut résoudre l'équation de Laplace (1.5) sous la condition (1.8). Pour cela, on cherche une solution sous forme de série, on suppose

On obtient

$$
\varphi(x, y, z, t)=\sum_{n=0}^{\infty} f_{n}(x, y, t) z^{n}
$$

$$
\varphi_{z}(x, y, z, t)=\sum_{n=1}^{\infty} n f_{n}(x, y, t) z^{n-1}
$$

d'où si $z=0$, on a $f_{1}(x, y, t)=0$. L'équation (1.5) s'écrit

$$
\begin{aligned}
\sum_{n=0}^{\infty} z^{n}\left(\beta \frac{\partial^{2} f_{n}}{\partial x^{2}}+\beta \gamma \frac{\partial^{2} f_{n}}{\partial y^{2}}\right)+\sum_{n=2}^{\infty} n(n-1) z^{n-2} f_{n} & =0 \\
\sum_{n=0}^{\infty}\left(\beta \frac{\partial^{2} f_{n}}{\partial x^{2}}+\beta \gamma \frac{\partial^{2} f_{n}}{\partial y^{2}}+(n+1)(n+2) f_{n+2}\right) z^{n} & =0
\end{aligned}
$$

Il s'ensuit que pour tout entier $n$,

$$
\beta \frac{\partial^{2} f_{n}}{\partial x^{2}}+\beta \gamma \frac{\partial^{2} f_{n}}{\partial y^{2}}+(n+1)(n+2) f_{n+2}=0
$$

On résout ces équations par récurrence sur $n$. Comme $f_{1}=0$, on en déduit que pour tout $n \in \mathbb{N}, f_{2 n+1}=0$.

Pour les indices pairs, on a

$$
\beta \frac{\partial^{2} f_{0}}{\partial x^{2}}+\beta \gamma \frac{\partial^{2} f_{0}}{\partial y^{2}}+2 f_{2}=0, \quad \beta \frac{\partial^{2} f_{2}}{\partial x^{2}}+\beta \gamma \frac{\partial^{2} f_{2}}{\partial y^{2}}+12 f_{4}=0
$$

ou encore

$$
f_{2}=-\left(\frac{\beta}{2} \frac{\partial^{2} f_{0}}{\partial x^{2}}+\frac{\beta \gamma}{2} \frac{\partial^{2} f_{0}}{\partial y^{2}}\right), \quad f_{4}=\left(\frac{\beta^{2}}{24} \frac{\partial^{4} f_{0}}{\partial x^{4}}+\frac{\beta^{2} \gamma+\beta \gamma^{2}}{24} \frac{\partial^{4} f_{0}}{\partial x^{2} \partial y^{2}}+\frac{\beta^{2} \gamma^{2}}{24} \frac{\partial^{4} f_{0}}{\partial y^{4}}\right)
$$


On note $f:=f_{0}$. On effectue alors un développement limité de $\varphi$ pour $\beta$ proche de zéro. On trouve

$$
\varphi(x, y, z, t)=f-\left(\frac{\beta}{2} f_{x x}+\frac{\beta \gamma}{2} f_{y y}\right) z^{2}+\left(\frac{\beta^{2}}{24} f_{x x x x}+\frac{\beta^{2} \gamma+\beta \gamma^{2}}{24} f_{x x y y}+\frac{\beta^{2} \gamma^{2}}{24} f_{y y y y}\right) z^{4}+O\left(\beta^{3}\right) .
$$

L'équation (1.7) devient pour $z=1+\alpha \eta$

$$
\begin{aligned}
\eta_{t}+\alpha\left[f_{x}-\left(\frac{\beta}{2} f_{x x x}+\frac{\beta \gamma}{2} f_{x y y}\right)(1+\alpha \eta)^{2}\right] \eta_{x} & +\alpha \gamma\left[f_{y}-\left(\frac{\beta}{2} f_{x x y}+\frac{\beta \gamma}{2} f_{y y y}\right)(1+\alpha \eta)^{2}\right] \eta_{y} \\
& +\left(f_{x x}+\gamma f_{y y}\right)(1+\alpha \eta)-\frac{\beta}{6} f_{x x x x}(1+\alpha \eta)^{3}=O\left(\alpha^{2}+\beta^{2}+\gamma^{2}\right),
\end{aligned}
$$

et plus simplement

$$
\eta_{t}+\left((1+\alpha \eta) f_{x}\right)_{x}-\frac{\beta}{6} f_{x x x x}+\gamma f_{y y}=O\left(\alpha^{2}+\beta^{2}+\gamma^{2}\right) .
$$

De la même manière, l'équation (1.6) devient pour $z=1+\alpha \eta$

$$
\begin{aligned}
& \eta+\left[f_{t}-\left(\frac{\beta}{2} f_{x x t}+\frac{\beta \gamma}{2} f_{y y t}\right)(1+\alpha \eta)^{2}\right] \\
& +\frac{\alpha}{2}\left[f_{x}-\left(\frac{\beta}{2} f_{x x x}+\frac{\beta \gamma}{2} f_{x y y}\right)(1+\alpha \eta)^{2}\right]^{2}+\frac{\alpha \gamma}{2}\left[f_{y}-\left(\frac{\beta}{2} f_{x x y}+\frac{\beta \gamma}{2} f_{y y y}\right)(1+\alpha \eta)^{2}\right]^{2} \\
& +\frac{\alpha}{\beta}\left[\left(\beta f_{x x}+\beta \gamma f_{y y}\right)(1+\alpha \eta)-\frac{\beta}{6} f_{x x x x}(1+\alpha \eta)^{3}\right]^{2}-\sigma \beta \eta_{x x}=O\left(\alpha^{2}+\beta^{2}+\gamma^{2}\right),
\end{aligned}
$$

et en simplifiant

$$
\eta+f_{t}+\frac{\alpha}{2} f_{x}^{2}-\beta\left(\frac{1}{2} f_{x x t}+\sigma \eta_{x x}\right)=O\left(\alpha^{2}+\beta^{2}+\gamma^{2}\right)
$$

On pose $w:=f_{x}$ et on dérive par rapport à $x$ les équations (1.9) et (1.10) pour avoir finalement deux équations de type Boussinesq

$$
\begin{aligned}
\eta_{t}+((1+\alpha \eta) w)_{x}-\frac{\beta}{6} w_{x x x}+\gamma f_{y y} & =O\left(\alpha^{2}+\beta^{2}+\gamma^{2}\right), \\
\eta_{x}+w_{t}+\alpha w w_{x}-\beta\left(\frac{1}{2} w_{x x t}+\sigma \eta_{x x x}\right) & =O\left(\alpha^{2}+\beta^{2}+\gamma^{2}\right) .
\end{aligned}
$$

À l'ordre le plus bas, les équations (1.11) et (1.12) deviennent

$$
\eta_{t}+w_{x}=O(\alpha+\beta) \text { et } \eta_{x}+w_{t}=O(\alpha+\beta),
$$

ce qui implique $\eta_{t t}-\eta_{x x}=O(\alpha+\beta)$. Si on suppose que les ondes se déplacent de la gauche vers la droite dans la direction $x$, la solution de cette équation est de la forme $\eta(x, y, t)=\eta(x-t, y, 0)+O(\alpha+\beta)$, ce qui implique $\eta_{t}+\eta_{x}=O(\alpha+\beta)$. On a donc

$$
\eta_{t}-w_{t}=\eta_{t}+\eta_{x}=O(\alpha+\beta) \text { et } \eta_{x}-w_{x}=\eta_{x}+\eta_{t}=O(\alpha+\beta),
$$

d'où $\eta=w$ à l'ordre le plus bas. Il nous faut alors corriger l'égalité au premier ordre $w=\eta$ en cherchant $w$ sous la forme

$$
w=\eta+\alpha A+\beta B+\gamma C+O\left(\alpha^{2}+\beta^{2}+\gamma^{2}\right),
$$

et on veut déterminer les valeurs de $A, B$, et $C$ au premier ordre. L'équation (1.11) devient

$$
\eta_{t}+\eta_{x}+\alpha\left(A_{x}+2 \eta \eta_{x}\right)+\beta\left(B_{x}-\frac{1}{6} \eta_{x x x}\right)+\gamma\left(C_{x}+f_{y y}\right)=O\left(\alpha^{2}+\beta^{2}+\gamma^{2}\right),
$$


et l'équation (1.12)

$$
\eta_{t}+\eta_{x}+\alpha\left(A_{t}+\eta \eta_{x}\right)+\beta\left(B_{t}-\frac{1}{2} \eta_{x x t}-\sigma \eta_{x x x}\right)+\gamma C_{t}=O\left(\alpha^{2}+\beta^{2}+\gamma^{2}\right)
$$

et comme $\eta_{t}=-\eta_{x}+O(\alpha+\beta+\gamma)$, on trouve

$$
\eta_{t}+\eta_{x}+\alpha\left(A_{t}+\eta \eta_{x}\right)+\beta\left(B_{t}+\frac{1}{2} \eta_{x x x}-\sigma \eta_{x x x}\right)+\gamma C_{t}=O\left(\alpha^{2}+\beta^{2}+\gamma^{2}\right) .
$$

La compatibilité des développements limités des équations (1.13) et (1.15) implique

$$
A_{x}+2 \eta \eta_{x}=A_{t}+\eta \eta_{x}, B_{x}-\frac{1}{6} \eta_{x x x}=B_{t}+\left(\frac{1}{2}-\sigma\right) \eta_{x x x} \text { et } C_{x}+f_{y y}=C_{t}
$$

On effectue le changement de variables $r=x-t$ et $s=x+t$ pour obtenir

$$
A=\frac{-\eta^{2}}{4}, B=\left(\frac{1}{3}-\frac{\sigma}{2}\right) \eta_{x x} \text { et } C_{r}=\frac{-1}{2} f_{y y}
$$

Remplacer ces valeurs dans (1.13) donne

$$
\eta_{t}+\eta_{x}+\frac{3}{2} \alpha \eta \eta_{x}+\left(\frac{1}{6}-\frac{\sigma}{2}\right) \beta \eta_{x x x}+\frac{\gamma}{2} f_{y y}=O\left(\alpha^{2}+\beta^{2}+\gamma^{2}\right)
$$

et comme $\eta_{t}=-\eta_{x}+O(\alpha+\beta+\gamma)$, on déduit de l'équation précédente

$$
\eta_{t}+\eta_{x}+\frac{3}{2} \alpha \eta \eta_{x}-\left(\frac{1}{6}-\frac{\sigma}{2}\right) \beta \eta_{x x t}+\frac{\gamma}{2} f_{y y}=O\left(\alpha^{2}+\beta^{2}+\gamma^{2}\right) .
$$

Finalement, on dérive par rapport à $x$ ces deux dernières équations pour trouver

$$
\begin{aligned}
& \left(\eta_{t}+\eta_{x}+\frac{3}{2} \alpha \eta \eta_{x}+\left(\frac{1}{6}-\frac{\sigma}{2}\right) \beta \eta_{x x x}\right)_{x}+\frac{\gamma}{2} \eta_{y y}=O\left(\alpha^{2}+\beta^{2}+\gamma^{2}\right) \\
& \left(\eta_{t}+\eta_{x}+\frac{3}{2} \alpha \eta \eta_{x}-\left(\frac{1}{6}-\frac{\sigma}{2}\right) \beta \eta_{x x t}\right)_{x}+\frac{\gamma}{2} \eta_{y y}=O\left(\alpha^{2}+\beta^{2}+\gamma^{2}\right) .
\end{aligned}
$$

Les équations (1.16) au premier ordre sont les équations de KP, appelées KP-I si $\sigma>1 / 3$, KP-II si $\sigma<1 / 3$, et les équations (1.17) au premier ordre sont les équations de KP-BBM, appelées KP-BBM-I si $\sigma>1 / 3$ et KP-BBM-II si $\sigma<1 / 3$. Ce problème est mal posé si $\sigma>1 / 3$, en effet on peut écrire l'équation précédente

$$
\left(1-\frac{\beta}{2}\left(\frac{1}{3}-\sigma\right) \partial_{x}^{2}\right) \eta_{t}=-\eta_{x}-\frac{3}{2} \alpha \eta \eta_{x}-\frac{\gamma}{2} \partial_{x}^{-1} \eta_{y y}
$$

où $\partial_{x}^{-1}$ désigne l'antidérivée dans la direction $x$, de symbole de Fourier $\mathcal{F}\left(\partial_{x}^{-1}\right)(k)=\frac{1}{i k}$. On remarque que l'opérateur $\left(1+c \partial_{x}^{2}\right)$, pour $c>0$, n'est pas inversible pour toute fonction $u$ de $L^{2}(\mathbb{R})$, car on a

$$
\left(1+c \partial_{x}^{2}\right) u(x)=\mathcal{F}^{-1}\left(\left(1-c k^{2}\right) \mathcal{F} u\right)(x),
$$

et $\left(1-c k^{2}\right)$ s'annule si $c>0$. On remarque de la même manière, qu'en dimension 1 , l'équation BBM est mal posée pour $\sigma>1 / 3$. 
On peut refaire la même démarche en supposant que la tension de surface $T$ est nulle, on retrouve alors les équations de KP-II et KP-BBM-II.

Maintenant qu'il est clair que les équations de KP-II et KP-BBM-II modélisent le même phénomène physique, il est naturel de vouloir les comparer.

\section{Comparaison des solutions de KP-II et KP-BBM-II}

On considère les problèmes de Cauchy suivants

$$
\begin{aligned}
\eta_{t}+\eta_{x}+\alpha \eta \eta_{x}+\alpha \eta_{x x x}+\alpha \partial_{x}^{-1} \eta_{y y} & =0 \\
\zeta_{t}+\zeta_{x}+\alpha \zeta \zeta_{x}-\alpha \zeta_{x x t}+\alpha \partial_{x}^{-1} \zeta_{y y} & =0 \\
\eta(x, y, 0)=\zeta(x, y, 0) & =f(x, y)
\end{aligned}
$$

où $\alpha=\beta=\gamma$ dans la modélisation précédente. L'équation (2.1) est l'équation de KP-II et l'équation (2.2) est l'équation de KP-BBM-II.

\subsection{Discussion linéaire}

On suppose que le problème précédent ne présente pas de non-linéarité

$$
\begin{aligned}
\eta_{t}+\eta_{x}+\alpha \eta_{x x x}+\alpha \partial_{x}^{-1} \eta_{y y} & =0 \\
\zeta_{t}+\zeta_{x}-\alpha \zeta_{x x t}+\alpha \partial_{x}^{-1} \zeta_{y y} & =0 \\
\eta(x, y, 0)=\zeta(x, y, 0) & =f(x, y)
\end{aligned}
$$

Supposons que la donnée initiale $f$ appartienne à la classe de Schwartz $\mathcal{S}\left(\mathbb{R}^{2}\right)$. Pour alléger les écritures, on notera par $\wedge$ la transformée de Fourier en espace et $\vee$ la transformée de Fourier inverse. Formellement, la transformée de Fourier en espace donne pour l'équation KP-II une équation différentielle ordinaire dont la solution est pour tout $t \in \mathbb{R}$ et $(k, l) \in \mathbb{R}^{2}$

$$
\hat{\eta}(k, l, t)=\exp \left(-i k\left(1-\alpha k^{2}+\alpha \frac{l^{2}}{k^{2}}\right) t\right) \hat{f}(k, l)
$$

De la même manière, la transformée de Fourier en espace donne pour l'équation KP-BBM-II une équation différentielle ordinaire dont la solution est pour tout $t \in \mathbb{R}$ et $(k, l) \in \mathbb{R}^{2}$

$$
\hat{\zeta}(k, l, t)=\exp \left(-i k\left(\frac{1+\alpha \frac{l^{2}}{k^{2}}}{1+\alpha k^{2}}\right) t\right) \hat{f}(k, l) .
$$

On a, pour tous réels $k, l$ et $t$,

$$
\begin{aligned}
\hat{\eta}(k, l, t)-\hat{\zeta}(k, l, t) & =\exp \left(-i k\left(\frac{\left.\left.1+\alpha \frac{l^{2}}{k^{2}}\right) t\right)}{1+\alpha k^{2}}\right)\right. \\
& \times\left(\exp \left(-i k\left(1-\alpha k^{2}+\alpha \frac{l^{2}}{k^{2}}\right) t\right) \exp \left(i k\left(\frac{1+\alpha \frac{l^{2}}{k^{2}}}{1+\alpha k^{2}}\right) t\right)-1\right) \hat{f}(k, l) .
\end{aligned}
$$


On déduit du fait que pour $x \in \mathbb{R},|\exp (i x)-1| \leq 2|x|$, que

$$
\begin{aligned}
|\hat{\eta}(k, l, t)-\hat{\zeta}(k, l, t)| & =\left|\exp \left(-i k\left(1-\alpha k^{2}+\alpha \frac{l^{2}}{k^{2}}\right) t\right) \exp \left(i k\left(\frac{1+\alpha \frac{l^{2}}{k^{2}}}{1+\alpha k^{2}}\right) t\right)-1\right||\hat{f}(k, l)| \\
& \leq 2\left|-k\left(1-\alpha k^{2}+\alpha \frac{l^{2}}{k^{2}}\right) t+k\left(\frac{1+\alpha \frac{l^{2}}{k^{2}}}{1+\alpha k^{2}}\right) t\right||\hat{f}(k, l)|,
\end{aligned}
$$

et en mettant au même dénominateur, on trouve

$$
|\hat{\eta}(k, l, t)-\hat{\zeta}(k, l, t)| \leq 2 \alpha^{2}|t| \frac{\left|-k^{5}+l^{2} k\right|}{1+\alpha k^{2}}|\hat{f}(k, l)| .
$$

Comme, pour tout $k \in \mathbb{R}, 1+\alpha k^{2} \geq 1$, on en déduit que

$$
|\hat{\eta}(k, l, t)-\hat{\zeta}(k, l, t)| \leq 2 \alpha^{2}|t|\left(\left|k^{5}\right|+\left|l^{2} k\right|\right)|\hat{f}(k, l)| .
$$

Soit $t \in \mathbb{R}$, l'inversion de Fourier implique que

$$
\begin{aligned}
\|\eta(., ., t)-\zeta(., ., t)\|_{\infty} & =\sup _{x, y \in \mathbb{R}}\left|\frac{1}{(2 \pi)^{2}} \int_{\mathbb{R}^{2}} \mathrm{e}^{i k x+i l y}(\hat{\eta}(k, l, t)-\hat{\zeta}(k, l, t)) \mathrm{d} k \mathrm{~d} l\right| \\
& \leq \frac{1}{(2 \pi)^{2}}\|\hat{\eta}(., ., t)-\hat{\zeta}(., ., t)\|_{L^{1}\left(\mathbb{R}^{2}\right)} .
\end{aligned}
$$

L'inégalité (2.4) donne finalement

$$
\|\eta(., ., t)-\zeta(., ., t)\|_{\infty} \leq\left(\frac{2}{(2 \pi)^{2}} \int_{\mathbb{R}^{2}}\left(\left|k^{5}\right|+\left|l^{2} k\right|\right)|\hat{f}(k, l)| \mathrm{d} k \mathrm{~d} l\right) \alpha^{2}|t| .
$$

Cette analyse du problème linéaire nous mène à la conjecture suivante :

Conjecture 2.1. Soit $f$ une fonction définie sur $\mathbb{R}^{2}$ telle que $\widehat{\partial_{x}^{5} f}$ et $\widehat{\partial_{x} \partial_{y}^{2} f}$ soient intégrables sur $\mathbb{R}^{2}$. Alors $\eta$ et $\zeta$, les solutions respectives de KP-II et KP-BBM-II, vérifient qu'il existe une constante $C>0$, qui dépend uniquement de $f$ et une constante $0<\delta<1$, telle que pour $|t| \leq \alpha^{-1-\delta}$, on ait

$$
\|\eta(., ., t)-\zeta(., ., t)\|_{\infty} \leq C \alpha^{2}|t| .
$$

On verra qu'une comparaison semblable peut être prouvée sur un intervalle de temps $\left[0, \alpha^{-1}\right]$ dans la Section 2.3. Néanmoins, on se demandera si, à l'aide d'une méthode numérique, on peut améliorer ce résultat et ainsi se rapprocher de la conjecture.

\subsection{Problèmes de Cauchy}

On rappelle tout d'abord les principales notations utilisées : On note $L^{2}\left(\mathbb{R}^{2}\right)$ l'espace des fonctions de carré intégrable muni de la norme

$$
\|f\|^{2}=\int_{\mathbb{R}^{2}}|f(x, y)|^{2} \mathrm{~d} x \mathrm{~d} y,
$$

et $L^{\infty}\left(\mathbb{R}^{2}\right)$ l'espace des fonctions bornées muni de la norme

$$
\|f\|_{\infty}=\sup \operatorname{ess}(f):=\inf \left\{c ;|f(x, y)| \leq c \text { presque partout dans } \mathbb{R}^{2}\right\} .
$$


Pour tout réel positif $m, H^{m}\left(\mathbb{R}^{2}\right)$ est l'espace de Sobolev des fonctions de carré intégrable, dont les $m$ premières dérivées sont aussi de carré intégrable, muni ici de la norme

$$
\|f\|_{m}^{2}=\int_{\mathbb{R}^{2}}\left(1+k^{2}+l^{2}\right)^{m}|\hat{f}(k, l)|^{2} \mathrm{~d} k \mathrm{~d} l
$$

On note $X^{m}\left(\mathbb{R}^{2}\right)$ l'ensemble des fonctions $f$ de $H^{m}\left(\mathbb{R}^{2}\right)$ telles que $\partial_{x}^{-1} f$ soit dans $H^{m}\left(\mathbb{R}^{2}\right)$, muni de la norme

$$
|f|_{m}=\left(\|f\|_{m}^{2}+\left\|\partial_{x}^{-1} f\right\|_{m}^{2}\right)^{1 / 2}
$$

L'espace $\mathcal{S}\left(\mathbb{R}^{2}\right)$ désigne la classe de Schwartz, et $\mathcal{S}^{\prime}\left(\mathbb{R}^{2}\right)$ celui des distributions tempérées.

Si $X$ est un espace de Banach quelconque, et $T>0$, l'espace $\mathcal{C}([-T, T] ; X)$ est l'ensemble des fonctions continues de $[-T, T]$ dans $X$, muni de la norme $\sup _{-T \leq t \leq T}\|f\|_{X}$, avec $\|\cdot\|_{X}$ une norme sur $X$.

On rappelle que les problèmes de Cauchy $(2.1)-(2.2)-(2.3)$ sont globalement bien posés en temps. Pour l'équation de KP-II, Bourgain [8] montre que pour tout donnée initiale prise dans $H^{m}\left(\mathbb{R}^{2}\right)$, avec $m \geq 0$, il existe une unique solution de (2.1)-(2.3) globale en temps. Le problème de Cauchy pour l'équation de KP-BBM-II est traité dans les articles de Bona et al. [7], ou Saut et Tzvetkov [18], ils montrent que pour toute donnée initiale prise dans le sous-espace de $L^{2}\left(\mathbb{R}^{2}\right)$ muni de la norme $\left(\|u\|^{2}+\left\|u_{x}\right\|^{2}\right)^{1 / 2}$, il existe une unique solution de $(2.2)-(2.3)$ globale en temps.

Nous n'aurons besoin dans ce travail que de l'existence et l'unicité du problème de Cauchy local. On effectue alors le changement de fonctions

$$
u(x, y, t)=\eta\left(x+\alpha^{-1} t, y, \alpha^{-1} t\right), v(x, y, t)=\zeta\left(x+\alpha^{-1} t, y, \alpha^{-1} t\right) .
$$

Le problème de Cauchy (2.1)-(2.2)-(2.3) devient

$$
\begin{aligned}
u_{t}+u u_{x}+u_{x x x}+\partial_{x}^{-1} u_{y y} & =0 \\
v_{t}+v v_{x}+v_{x x x}-\alpha v_{x x t}+\partial_{x}^{-1} v_{y y} & =0 \\
u(x, y, 0)=v(x, y, 0) & =f(x, y) .
\end{aligned}
$$

Pour le problème de Cauchy (2.5)-(2.7), Iório et Nunes [12] nous donnent la condition suivante :

Proposition 2.2. Soit $f \in X^{m}\left(\mathbb{R}^{2}\right), m>2$. Alors il existe une constante $C_{m}>0$, dépendant uniquement de $m$, telle que pour $T_{0}:=\frac{1}{C_{m}|f|_{m}}$, il existe une unique solution $u \in \mathcal{C}\left(\left[-T_{0}, T_{0}\right] ; X^{m}\left(\mathbb{R}^{2}\right)\right)$ du problème de Cauchy (2.5)-(2.7). De plus, cette solution vérifie qu'il existe une constante $c_{m}>0$ telle que pour $|t| \leq T_{0}$, on $a|u(t)|_{m} \leq c_{m}|f|_{m}$.

On utilise la méthode décrite dans l'article de Iório et Nunes [12] pour trouver un résultat analogue pour le problème de Cauchy (2.6)-(2.7).

Proposition 2.3. Soit $f \in X^{m}\left(\mathbb{R}^{2}\right), m>2$. Alors il existe une constante $C_{m}>0$, dépendant uniquement de $m$, telle que pour $T_{0}:=\frac{1}{C_{m}|f|_{m}}$, il existe une unique solution $v \in \mathcal{C}\left(\left[-T_{0}, T_{0}\right] ; X^{m}\left(\mathbb{R}^{2}\right)\right)$ du problème de Cauchy (2.6)-(2.7). De plus, cette solution vérifie qu'il existe une constante $c_{m}>0$ telle que pour $|t| \leq T_{0}$, on $a|v(t)|_{m} \leq c_{m}|f|_{m}$

Il paraît évident que le temps d'existence local pour le problème de Cauchy (2.5)-(2.7) soit indépendant de $\alpha$, il l'est moins pour le problème de Cauchy (2.6)-(2.7).

Lemme 2.4. Soit $m>2$. Il existe une constante $C_{m}>0$, dépendant uniquement de $m$, telle que pour tout $v \in H^{m}\left(\mathbb{R}^{2}\right)$

$$
\left|\left\langle v, v v_{x}\right\rangle_{m}\right| \leq C_{m}\|v\|_{m}^{3}
$$


où le produit scalaire est défini par, pour $f$ et $g$ appartenant à $H^{m}\left(\mathbb{R}^{2}\right)$,

$$
\langle f, g\rangle_{m}:=\int_{\mathbb{R}^{2}}\left(1+k^{2}+l^{2}\right)^{m} \hat{f}(k, l) \overline{\hat{g}(k, l)} \mathrm{d} k \mathrm{~d} l .
$$

Démonstration. On définit l'opérateur $J^{m}$ par, pour tout $f \in \mathcal{S}^{\prime}\left(\mathbb{R}^{2}\right)$,

$$
\widehat{J^{m}(f)}(k, l):=\left(1+k^{2}+l^{2}\right)^{m / 2} \hat{f}(k, l)
$$

En particulier, pour $f \in \mathcal{S}\left(\mathbb{R}^{2}\right)$, on a

$$
J^{m}(f)(x, y)=\frac{1}{(2 \pi)^{2}} \int_{\mathbb{R}^{2}} \mathrm{e}^{i(x k+y l)}\left(1+k^{2}+l^{2}\right)^{m / 2} \hat{f}(k, l) \mathrm{d} k \mathrm{~d} l .
$$

Kato et Ponce [14] montrent qu'il existe une constante $C_{m}>0$ telle que pour $f$ et $g$ appartenant à $\mathcal{S}\left(\mathbb{R}^{2}\right)$, on ait

$$
\left\|\left[J^{m}, f\right](g)\right\| \leq C_{m}\left(\|f\|_{\infty}\left\|J^{m-1} g\right\|+\left\|J^{m} f\right\|\|g\|_{\infty}\right),
$$

où le crochet est défini par $\left[J^{m}, f\right](g)=J^{m}(f g)-f J^{m}(g)$.

On remarque tout d'abord que pour $f$ fonction réelle, $J^{m}(f)$ est aussi réel, en effet, on a

$$
\begin{aligned}
\overline{J^{m}(f)(x, y)} & =\frac{1}{(2 \pi)^{2}} \int_{\mathbb{R}^{2}} \mathrm{e}^{-i(x k+y l)}\left(1+k^{2}+l^{2}\right)^{m / 2} \overline{\hat{f}(k, l)} \mathrm{d} k \mathrm{~d} l \\
& =\frac{1}{(2 \pi)^{2}} \int_{\mathbb{R}^{2}} \mathrm{e}^{-i(x k+y l)}\left(1+k^{2}+l^{2}\right)^{m / 2}\left(\int_{\mathbb{R}^{2}} \mathrm{e}^{i(\widetilde{x} k+\widetilde{y} l)} f(\widetilde{x}, \widetilde{y}) \mathrm{d} \widetilde{x} \mathrm{~d} \widetilde{y}\right) \mathrm{d} k \mathrm{~d} l,
\end{aligned}
$$

le changement de variable $(k, l) \rightarrow(-k,-l)$, implique que $\overline{J^{m}(f)}=J^{m}(f)$.

Supposons que $v$ appartienne à $\mathcal{S}\left(\mathbb{R}^{2}\right)$, on a alors

$$
\left\langle v, v v_{x}\right\rangle_{m}=\int_{\mathbb{R}^{2}} J^{m}(v) \overline{J^{m}\left(v v_{x}\right)}=\int_{\mathbb{R}^{2}} J^{m}(v) J^{m}\left(v v_{x}\right) .
$$

Or, $J^{m}\left(v v_{x}\right)=v J^{m}\left(v_{x}\right)+\left[J^{m}, v\right]\left(v_{x}\right)$, d'où

$$
\left\langle v, v v_{x}\right\rangle_{m}=\int_{\mathbb{R}^{2}} v J^{m}\left(v_{x}\right) J^{m}(v)+\int_{\mathbb{R}^{2}}\left[J^{m}, v\right]\left(v_{x}\right) J^{m}(v) .
$$

Majorons ces deux intégrales : d'une part

$$
\int_{\mathbb{R}^{2}} v J^{m}\left(v_{x}\right) J^{m}(v)=\frac{1}{2} \int_{\mathbb{R}^{2}} v\left(J^{m}(v)^{2}\right)_{x}
$$

une intégration par parties donne

$$
\left|\int_{\mathbb{R}^{2}} v J^{m}\left(v_{x}\right) J^{m}(v)\right|=\left|-\frac{1}{2} \int_{\mathbb{R}^{2}} v_{x} J^{m}(v)^{2}\right| \leq \frac{1}{2}\left\|v_{x}\right\|_{\infty}\left\|J^{m} v\right\|^{2},
$$

et l'injection de Sobolev implique qu'il existe une constante $C_{m}>0$ telle que

$$
\left|\int_{\mathbb{R}^{2}} v J^{m}\left(v_{x}\right) J^{m}(v)\right| \leq C_{m}\|v\|_{m}^{3} .
$$


D'autre part, l'inégalité de Cauchy-Schwarz donne

$$
\left|\int_{\mathbb{R}^{2}}\left[J^{m}, v\right]\left(v_{x}\right) J^{m}(v)\right| \leq\left\|\left[J^{m}, v\right] v_{x}\right\|\left\|J^{m} v\right\| .
$$

D'après l'inégalité $(2.8)$ avec $f=v$ et $g=v_{x}$, on trouve

$$
\left|\int_{\mathbb{R}^{2}}\left[J^{m}, v\right]\left(v_{x}\right) J^{m}(v)\right| \leq C_{m}\left(\|v\|_{\infty}\left\|J^{m-1} v_{x}\right\|+\left\|J^{m} v\right\|\left\|v_{x}\right\|_{\infty}\right)\left\|J^{m} v\right\|,
$$

et l'injection de Sobolev implique

$$
\left|\int_{\mathbb{R}^{2}}\left[J^{m}, v\right]\left(v_{x}\right) J^{m}(v)\right| \leq C_{m}\|v\|_{m}^{3}
$$

On généralise ce résultat aux fonctions de $H^{m}\left(\mathbb{R}^{2}\right)$ par densité de l'espace $\mathcal{S}\left(\mathbb{R}^{2}\right)$ dans les espaces de Sobolev.

On considère tout d'abord l'équation linéaire

$$
v_{t}+v_{x x x}-\alpha v_{x x t}+\partial_{x}^{-1} v_{y y}=0
$$

On pose alors pour $v \in X^{m}\left(\mathbb{R}^{2}\right)$

$$
A_{0} v:=\left(-i\left(\frac{k^{3}-l^{2} / k}{1+\alpha k^{2}}\right) \hat{v}(k, l)\right)^{\vee} .
$$

On remarque ([12], Lem. 4.1) que pour $v$ appartenant à $X^{m}\left(\mathbb{R}^{2}\right)$, on a l'égalité $v_{t}+A_{0} v=0$ dans $H^{m-3}\left(\mathbb{R}^{2}\right)$. Revenons à l'équation avec non-linéarité. On effectue maintenant une régularisation parabolique de cette équation, c'est-à-dire que l'on considère le problème

$$
\begin{aligned}
v_{t}+A_{\mu} v+a(v) & =0 \\
v(x, y, 0) & =f(x, y),
\end{aligned}
$$

où $A_{\mu}:=-\mu \Delta+A_{0}$, pour $\mu \geq 0, a(v):=\left(\frac{i k}{2\left(1+\alpha k^{2}\right)} \widehat{v^{2}}\right)^{\vee}$ et $f \in X^{m}\left(\mathbb{R}^{2}\right)$. On pose $E_{\mu}(t):=\exp \left(-t A_{\mu}\right)$.

Lemme 2.5. Soient $m$ et $n$ deux réels positifs. Alors il existe une constante $K_{n}>0$, dépendant uniquement de $n$, telle que pour tout $v \in X^{m}\left(\mathbb{R}^{2}\right)$ et pour tout temps $t \geq 0$, on ait

$$
\left|E_{\mu}(t) v\right|_{m+n} \leq K_{n}\left(1+\left(\frac{1}{2 \mu t}\right)^{n}\right)^{1 / 2}|v|_{m}
$$

Démonstration. La preuve est la même que [11], Lemme 1.1.

Lemme 2.6. Soit $m>2$. Il existe un temps $T$, dépendant de $\mu$, tel que sur la boule fermée

$$
\bar{B}_{m}(T)=\left\{v \in \mathcal{C}\left([0, T] ; X^{m}\left(\mathbb{R}^{2}\right)\right) ;\left|E_{\mu}(t) f-v(t)\right|_{m} \leq|f|_{m}\right\},
$$

l'application

soit une contraction.

$$
\Phi(v)(t):=E_{\mu}(t) f-\int_{0}^{t} E_{\mu}(t-\tau) a(v)(\tau) \mathrm{d} \tau
$$

De plus, il existe une constante $C_{m}>0$, indépendant de $\mu$, telle que la solution $v$ de $(2.12)$, définie sur [0,T], se prolonge de manière unique sur $\left[0, T_{0}\right]$, avec $T_{0}=\frac{1}{C_{m}|f|_{m}}$. 
Démonstration. Soient $T>0$, et $u, v$ deux éléments de $\bar{B}_{m}(T)$, on a pour $t \in[0, T]$, d'après l'inégalité de Minkowski,

$$
\begin{aligned}
|\Phi(v)(t)-\Phi(u)(t)|_{m} & =\left|\int_{0}^{t} E_{\mu}(t-\tau) a(u)(\tau)-E_{\mu}(t-\tau) a(v)(\tau) \mathrm{d} \tau\right|_{m} \\
& \leq \int_{0}^{t}\left|E_{\mu}(t-\tau) a(u)(\tau)-E_{\mu}(t-\tau) a(v)(\tau)\right|_{m} \mathrm{~d} \tau .
\end{aligned}
$$

En écrivant $m=m-1+1$, l'inégalité (2.11) donne

$$
|\Phi(v)(t)-\Phi(u)(t)|_{m} \leq K_{1} \int_{0}^{t}\left(1+\frac{1}{2 \mu(t-\tau)}\right)^{1 / 2}|a(v)(\tau)-a(u)(\tau)|_{m-1} \mathrm{~d} \tau
$$

On remarque que

$$
\begin{aligned}
\|a(v)-a(u)\|_{m-1}^{2} & =\int_{\mathbb{R}^{2}}\left(1+k^{2}+l^{2}\right)^{m-1}\left|\frac{i k}{2\left(1+\alpha k^{2}\right)}\left(\widehat{v^{2}}-\widehat{u^{2}}\right)\right|^{2} \mathrm{~d} k \mathrm{~d} l \\
& \leq \int_{\mathbb{R}^{2}}\left(1+k^{2}+l^{2}\right)^{m-1}|k|^{2}\left|\widehat{v^{2}}-\widehat{u^{2}}\right|^{2} \mathrm{~d} k \mathrm{~d} l \\
& \leq \int_{\mathbb{R}^{2}}\left(1+k^{2}+l^{2}\right)^{m}|(v-\widehat{u)(v}+u)|^{2} \mathrm{~d} k \mathrm{~d} l \leq\|(v+u)(v-u)\|_{m}^{2},
\end{aligned}
$$

et comme $m>2$, il existe une constante $C_{m}>0$ telle que

$$
\|a(v)-a(u)\|_{m-1}^{2} \leq C_{m}^{2}\left(\|u\|_{m}+\|v\|_{m}\right)^{2}\|v-u\|_{m}^{2} .
$$

Plus généralement, on montre

$$
|a(v)-a(u)|_{m-1} \leq C_{m}\left(|u|_{m}+|v|_{m}\right)|v-u|_{m} .
$$

Finalement

$$
|\Phi(v)(t)-\Phi(u)(t)|_{m} \leq C_{m} K_{1} \int_{0}^{t}\left(1+\frac{1}{2 \mu(t-\tau)}\right)^{1 / 2}\left(|u|_{m}+|v|_{m}\right)(\tau)|v-u|_{m}(\tau) \mathrm{d} \tau .
$$

Or, pour $v$ appartenant à la boule $\bar{B}_{m}(T)$, on a d'après l'inégalité (2.11),

$$
|v|_{m} \leq\left|E_{\mu}(t)(f)-v(t)\right|_{m}+\left|E_{\mu}(t)(f)\right|_{m} \leq\left(1+\sqrt{2} K_{0}\right)|f|_{m}
$$

d'où

$$
|\Phi(v)(t)-\Phi(u)(t)|_{m} \leq 2 C_{m} K_{1}\left(1+\sqrt{2} K_{0}\right)|f|_{m} \int_{0}^{t}\left(1+\frac{1}{2 \mu(t-\tau)}\right)^{1 / 2} \mathrm{~d} \tau\left(\sup _{\tau \in[0, t]}|v-u|_{m}(\tau)\right) .
$$

Comme, pour $a$ et $b$ positifs, $\sqrt{a+b} \leq \sqrt{a}+\sqrt{b}$, on a aussi

$$
\int_{0}^{t}\left(1+\frac{1}{2 \mu(t-\tau)}\right)^{1 / 2} \mathrm{~d} \tau \leq \int_{0}^{\infty} 1+\left(\frac{1}{2 \mu(t-\tau)}\right)^{1 / 2} \mathrm{~d} \tau \leq t+\frac{t^{1 / 2}}{\mu}
$$


Finalement, on a

$$
\sup _{t \in[0, T]}|\Phi(v)(t)-\Phi(u)(t)|_{m} \leq C_{m} K_{1}\left(1+\sqrt{2} K_{0}\right)\left(T+\frac{T^{1 / 2}}{2 \mu}\right)|f|_{m} \sup _{t \in[0, T]}|v-u|_{m}(t) .
$$

Pour conclure sur l'existence d'un temps $T$ telle que l'application $\Phi$ soit contractante, il suffit d'écrire que l'image de la boule fermée $\bar{B}_{m}(T)$ par $\Phi$ est incluse dans $\bar{B}_{m}(T)$ :

$$
\begin{aligned}
\left|E_{\mu}(f)(t)-\Phi(v)(t)\right|_{m} & =|\Phi(0)(t)-\Phi(v)(t)|_{m} \\
& \leq C_{m} K_{1}\left(1+\sqrt{2} K_{0}\right)\left(T+\frac{T^{1 / 2}}{2 \mu}\right)|f|_{m}^{2} \\
& \leq|f|_{m}
\end{aligned}
$$

et il faut choisir $T>0$ de telle sorte que $\left(T+\frac{T^{1 / 2}}{2 \mu}\right)<\frac{1}{C_{m} K_{1}\left(1+\sqrt{2} K_{0}\right)|f|_{m}}$.

Reste à montrer que l'on peut prolonger la solution de $(2.12)$ sur $\left[0, T_{0}\right]$, où ce temps $T_{0}$ peut être choisi indépendamment de $\mu$. Soit $v^{\mu}$ une solution de (2.9)-(2.10), on a

$$
\begin{aligned}
\partial_{t}\left\|v^{\mu}\right\|_{m}^{2} & =2\left\langle v^{\mu}, v_{t}^{\mu}\right\rangle_{m} \\
& =-2\left\langle v^{\mu}, A_{\mu} v^{\mu}\right\rangle_{m}-2\left\langle v^{\mu}, a\left(v^{\mu}\right)\right\rangle_{m} \\
& =2\left\langle v^{\mu}, \mu \Delta v^{\mu}\right\rangle_{m}-2\left\langle v^{\mu}, A_{0} v^{\mu}\right\rangle_{m}-2\left\langle v^{\mu}, a\left(v^{\mu}\right)\right\rangle_{m}
\end{aligned}
$$

Le premier produit scalaire est négatif; en effet on a

$$
2\left\langle v^{\mu}, \mu \Delta v^{\mu}\right\rangle_{m}=2 \int_{\mathbb{R}^{2}}\left(1+k^{2}+l^{2}\right)^{m} \hat{v}^{\mu} \overline{\overline{\mu \Delta v^{\mu}}} \mathrm{d} k \mathrm{~d} l=-2 \mu \int_{\mathbb{R}^{2}}\left(1+k^{2}+l^{2}\right)^{m}\left(k^{2}+l^{2}\right)\left|\hat{v}^{\mu}\right|^{2} \mathrm{~d} k \mathrm{~d} l .
$$

Pour le second produit scalaire, on a

$$
\begin{aligned}
-2\left\langle v^{\mu}, A_{0} v^{\mu}\right\rangle_{m} & =2 \int_{\mathbb{R}^{2}}\left(1+k^{2}+l^{2}\right)^{m} \hat{v}^{\mu}(k, l) \overline{\left(\frac{k^{3}-l^{2} / k}{1+\alpha k^{2}}\right) \hat{v^{\mu}}(k, l)} \mathrm{d} k \mathrm{~d} l \\
& =-2 i \int_{\mathbb{R}^{2}}\left(1+k^{2}+l^{2}\right)^{m} \hat{v}^{\mu}(k, l) \hat{v}^{\mu}(-k,-l)\left(\frac{k^{3}-l^{2} / k}{1+\alpha k^{2}}\right) \mathrm{d} k \mathrm{~d} l
\end{aligned}
$$

car, la fonction $v^{\mu}$ étant réelle, on a $\overline{\hat{v}^{\mu}(k, l, t)}=\hat{v}^{\mu}(-k,-l, t)$, et le changement de variables $(k, l) \rightarrow(-k,-l)$ implique que l'intégrale est nulle. On en déduit, via le Lemme 2.4, que, pour $m>2$, il existe une constante $C_{m}>0$ dépendant uniquement de $m$ telle que

$$
\partial_{t}\left\|\left.v^{\mu}\right|_{m} ^{2} \leq 2\left|\left\langle v^{\mu}, v^{\mu} v_{x}^{\mu}\right\rangle_{m}\right| \leq 2 C_{m}|| v^{\mu}\right\|_{m}^{3} \leq 2 C_{m}\left|v^{\mu}\right|_{m}^{3} .
$$

De la même manière, on trouve

$$
\partial_{t}\left\|\partial_{x}^{-1} v^{\mu}\right\|_{m}^{2} \leq 2\left|\left\langle\partial_{x}^{-1} v^{\mu}, \partial_{x}^{-1}\left(v^{\mu} v_{x}^{\mu}\right)\right\rangle_{m}\right| \leq 2 C_{m}\left|v^{\mu}\right|_{m}^{3}
$$

Finalement, on obtient

ou encore

$$
\partial_{t}\left|v^{\mu}(t)\right|_{m}^{2} \leq 2 C_{m}\left|v^{\mu}(t)\right|_{m}^{3}
$$

$$
\left|v^{\mu}(t)\right|_{m}^{2} \leq|f|_{m}^{2}+2 C_{m} \int_{0}^{t}\left|v^{\mu}(\tau)\right|_{m}^{3} \mathrm{~d} \tau
$$


Il s'ensuit que $\left|v^{\mu}(t)\right|_{m}^{2} \leq \rho(t)$, avec $\rho$ solution de

$$
\begin{aligned}
\partial_{t} \rho(t) & =2 C_{m} \rho^{3 / 2}(t) \\
\rho(0) & =|f|_{m}^{2} .
\end{aligned}
$$

D'où $\rho(t)^{1 / 2}=\frac{\rho(0)^{1 / 2}}{1-C_{m} \rho(0)^{1 / 2} t}$. On en déduit $\left|v^{\mu}(t)\right|_{m} \leq \frac{|f|_{m}}{1-C_{m}|f|_{m} t}$, et $v^{\mu}$ peut être prolongée en temps à l'intervalle $0 \leq t \leq T_{0}$ avec $T_{0}=\frac{1}{2 C_{m}|f|_{m}}$

Démonstration de la Proposition 2.3. On remarque tout d'abord que résoudre le problème de Cauchy (2.9)(2.10) revient à trouver un point fixe de l'application (2.12). En effet, soit $v$ vérifiant (2.9)-(2.10), et posons $w(t):=\mathrm{e}^{t A_{\mu}} v(t)$, on a

$$
w_{t}(t)=A_{\mu} \mathrm{e}^{t A_{\mu}} v(t)+\mathrm{e}^{t A_{\mu}} v_{t}(t)=A_{\mu} \mathrm{e}^{t A_{\mu}} v(t)-A_{\mu} \mathrm{e}^{t A_{\mu}} v(t)-\mathrm{e}^{t A_{\mu}} a(v)(t)=-\mathrm{e}^{t A_{\mu}} a(v)(t) .
$$

Or, $w(t)=w(0)+\int_{0}^{t} w_{t}(\tau) \mathrm{d} \tau$, d'où

$$
v(t)=\mathrm{e}^{-t A_{\mu}} f-\int_{0}^{t} \mathrm{e}^{-(t-\tau) A_{\mu}} a(v)(\tau) \mathrm{d} \tau
$$

Il suffit de voir si la limite lorsque $\mu$ tend vers 0 de $v^{\mu}$ existe sur l'intervalle de temps $\left[0, T_{0}\right]$. Soient $\mu$ et $\nu$ deux réels positifs, $v^{\mu}$ et $u^{\nu}$ les solutions respectives de (2.9)-(2.10). De la même manière que précédemment, on a pour $t \in\left[0, T_{0}\right]$

$$
\begin{aligned}
\partial_{t}\left\|v^{\mu}-u^{\nu}\right\|^{2} & =2\left\langle v^{\mu}-u^{\nu}, v_{t}^{\mu}-u_{t}^{\nu}\right\rangle \\
& =-2\left\langle v^{\mu}-u^{\nu}, A_{\mu} v^{\mu}-A_{\nu} u^{\nu}\right\rangle-2\left\langle v^{\mu}-u^{\nu}, a\left(v^{\mu}\right)-a\left(u^{\nu}\right)\right\rangle \\
& =2\left\langle v^{\mu}-u^{\nu}, \mu \Delta v^{\mu}-\nu \Delta u^{\nu}\right\rangle-2\left\langle v^{\mu}-u^{\nu}, A_{0} v^{\mu}-A_{0} u^{\nu}\right\rangle-2\left\langle v^{\mu}-u^{\nu}, a\left(v^{\mu}\right)-a\left(u^{\nu}\right)\right\rangle .
\end{aligned}
$$

L'opérateur $A_{0}$ étant linéaire, on a vu précédemment que

$$
\left\langle v^{\mu}-u^{\nu}, A_{0}\left(v^{\mu}-u^{\nu}\right)\right\rangle=0 .
$$

Pour le premier produit scalaire, on a

$$
2\left\langle v^{\mu}-u^{\nu}, \mu \Delta v^{\mu}-\nu \Delta u^{\nu}\right\rangle=2(\mu-\nu)\left\langle v^{\mu}-u^{\nu}, \Delta v^{\mu}\right\rangle+2 \nu\left\langle v^{\mu}-u^{\nu}, \Delta\left(v^{\mu}-u^{\nu}\right)\right\rangle
$$

Le fait que, pour $t \in\left[0, T_{0}\right], v^{\mu}(t)$ et $u^{\nu}(t)$ appartiennent à $X^{m}\left(\mathbb{R}^{2}\right)$, implique que ces fonctions et leurs dérivées d'ordre inférieur à $m$ tendent vers 0 à l'infini. Une intégration par parties implique alors que

$$
\left\langle v^{\mu}-u^{\nu}, \Delta\left(v^{\mu}-u^{\nu}\right)\right\rangle \leq 0
$$

On en déduit que

$$
\partial_{t}|| v^{\mu}-u^{\nu} \|^{2} \leq 2|\mu-\nu|\left|\left\langle v^{\mu}-u^{\nu}, \Delta v^{\mu}\right\rangle\right|+2\left|\left\langle v^{\mu}-u^{\nu}, a\left(v^{\mu}\right)-a\left(u^{\nu}\right)\right\rangle\right| .
$$

L'inégalité de Cauchy-Schwarz, puis l'inégalité triangulaire impliquent que

$$
\left|\left\langle v^{\mu}-u^{\nu}, \Delta v^{\mu}\right\rangle\right| \leq\left\|v^{\mu}-u^{\nu}\right\|_{1}|| v^{\mu} \|_{1} \leq\left(\left|v^{\mu}\right|_{m}+\left|u^{\nu}\right|_{m}\right)\left|v^{\mu}\right|_{m}
$$


Comme $\left|v^{\mu}\right|_{m} \leq \rho^{1 / 2}(t)$ et $\left|u^{\nu}\right|_{m} \leq \rho^{1 / 2}(t)$, on trouve pour le premier produit scalaire, si on pose $M=\sup _{t \in\left[0, T_{0}\right]} \rho(t)$,

$$
2|\mu-\nu|\left|\left\langle v^{\mu}-u^{\nu}, \Delta v^{\mu}\right\rangle\right| \leq 4 M|\mu-\nu| .
$$

Pour le second produit scalaire, on a

$$
2\left|\left\langle v^{\mu}-u^{\nu}, a\left(v^{\mu}\right)-a\left(u^{\nu}\right)\right\rangle\right| \leq 2\left|\left\langle v^{\mu}-u^{\nu}, v^{\mu} v_{x}^{\mu}-u^{\nu} u_{x}^{\nu}\right\rangle\right|
$$

et deux intégrations par parties donnent

$$
2\left|\left\langle v^{\mu}-u^{\nu}, a\left(v^{\mu}\right)-a\left(u^{\nu}\right)\right\rangle\right| \leq \frac{\left\|v_{x}^{\mu}+u_{x}^{\nu}\right\|_{\infty}}{2}\left\|v^{\mu}-u^{\nu}\right\|^{2} .
$$

L'injection de Sobolev implique qu'il existe une constante $C_{m}>0$ telle que

$$
2\left|\left\langle v^{\mu}-u^{\nu}, a\left(v^{\mu}\right)-a\left(u^{\nu}\right)\right\rangle\right| \leq C_{m} M^{1 / 2}\left\|v^{\mu}-u^{\nu}\right\|^{2} .
$$

On obtient finalement

$$
\partial_{t}\left\|v^{\mu}-u^{\nu}\right\|^{2} \leq 4 M|\mu-\nu|+C_{m} M^{1 / 2}\left\|v^{\mu}-u^{\nu}\right\|^{2} .
$$

Le lemme de Gronwall implique alors que, pour $t \in\left[0, T_{0}\right]$, la suite $\left(v_{\mu}(t)\right)_{\mu}$ est de Cauchy dans un espace complet, donc elle converge vers $v(t)=\lim _{\mu \rightarrow 0} v^{\mu}(t)$ dans $L^{2}\left(\mathbb{R}^{2}\right)$ et est unique. De plus, d'après le lemme précédent, l'application qui à $t \in\left[0, T_{0}\right]$ associe $v_{\mu}(t)$ solution de (2.9)-(2.10), est continue et uniformément bornée dans $L^{2}$ par $M^{1 / 2}$. En particulier, la suite $\left(v_{\mu}(t)\right)_{\mu}$ converge faiblement dans $X^{m}\left(\mathbb{R}^{2}\right)$ vers $v(t)$, pour tout $t \in\left[0, T_{0}\right]$, et l'application qui à $t \in\left[0, T_{0}\right]$ associe $v(t)$ est faiblement continue et uniformément bornée par $M^{1 / 2}$. En effet, en prenant $\mu$ suffisamment petit, pour tout $\varepsilon>0$, il existe $\delta>0$ tel que si $|t-s|<\delta$, alors pour toute fonction $\varphi \in \mathcal{S}\left(\mathbb{R}^{2}\right)$, on a

$$
\left|\langle v(t)-v(s), \varphi\rangle_{m}\right| \leq\left|\left\langle v(t)-v^{\mu}(t), \varphi\right\rangle_{m}\right|+\left|\left\langle v^{\mu}(t)-v^{\mu}(s), \varphi\right\rangle_{m}\right|+\left|\left\langle v^{\mu}(s)-v(s), \varphi\right\rangle_{m}\right| \leq 3 \varepsilon .
$$

Pour déduire la continuité forte, à savoir que $v$ appartienne à $\mathcal{C}\left(\left[0, T_{0}\right] ; X^{m}\left(\mathbb{R}^{2}\right)\right)$, on utilise l'argument de BonaSmith [5]. On peut alors conclure que $v$ est solution du problème de Cauchy $(2.6)-(2.7)$ dans $\mathcal{C}\left(\left[0, T_{0}\right] ; X^{m}\left(\mathbb{R}^{2}\right)\right)$. Pour les temps négatifs, il suffit de considérer dans les preuves précédentes $\mu \leq 0$, ce qui équivaut au cas où le temps est positif, ainsi que $\mu$.

Le fait que le temps $T_{0}$, donné par la proposition précédente, soit une constante indépendante de $\alpha$ est crucial; en effet on verra que le passage de $u$ à $\eta$ et de $v$ à $\zeta$ implique que si le temps $T_{0}$ est d'ordre 1 pour les solutions $u$ et $v$, alors il sera d'ordre $\alpha^{-1}$ pour les solutions $\eta$ et $\zeta$, à cela s'ajoute le fait que $\alpha$ est petit.

On aura aussi besoin de deux autres inégalités :

Lemme 2.7. Soient $m \geq 0$, et $u \in H^{m}\left(\mathbb{R}^{2}\right)$. On a

$$
\sup _{x, y \in \mathbb{R}^{2}}|u(x, y)| \leq 2\left(\|u\|\left\|u_{x}\right\|\left\|u_{y}\right\|\left\|u_{x y}\right\|\right)^{1 / 4} .
$$

Démonstration. Soit $u$ une fonction de $\mathcal{S}\left(\mathbb{R}^{2}\right)$ et $(x, y) \in \mathbb{R}^{2}$, on a

$$
|u(x, y)|^{2}=\int_{-\infty}^{x}\left(u^{2}(\widetilde{x}, y)\right)_{x} \mathrm{~d} \widetilde{x}=2 \int_{-\infty}^{x} u(\widetilde{x}, y) u_{x}(\widetilde{x}, y) \mathrm{d} \widetilde{x}
$$


et l'inégalité de Cauchy-Schwarz implique

$$
|u(x, y)|^{2} \leq 2\left(\int_{-\infty}^{+\infty} u^{2}(\widetilde{x}, y) \mathrm{d} \widetilde{x}\right)^{1 / 2}\left(\int_{-\infty}^{+\infty} u_{x}^{2}(\widetilde{x}, y) \mathrm{d} \widetilde{x}\right)^{1 / 2} .
$$

Il suffit de recommencer la même démarche en remarquant que

$$
|u(\widetilde{x}, y)|^{2}=\int_{-\infty}^{y}\left(u^{2}(\widetilde{x}, \widetilde{y})\right)_{y} \mathrm{~d} \widetilde{y} \text { et }\left|u_{x}(\widetilde{x}, y)\right|^{2}=\int_{-\infty}^{y}\left(u_{x}^{2}(\widetilde{x}, \widetilde{y})\right)_{y} \mathrm{~d} \widetilde{y} .
$$

On conclut en utilisant la densité des fonctions $\mathcal{S}\left(\mathbb{R}^{2}\right)$ dans les espaces de Sobolev.

De la même manière que pour le lemme de Gronwall, on montre :

Lemme 2.8. Soient $f$ et $g$ deux fonctions continues de $\left[0, T_{0}\right]$ dans $\mathbb{R}$, et c un réel tels que pour tout $t \in\left[0, T_{0}\right]$, on ait

$$
f(t) \leq g(t)+c \int_{0}^{t} f(\tau) \mathrm{d} \tau
$$

Alors pour tout $t \in\left[0, T_{0}\right]$, on a

$$
f(t) \leq g(t)+c \mathrm{e}^{c t} \int_{0}^{t} g(\tau) \mathrm{e}^{-c \tau} \mathrm{d} \tau
$$

On a désormais tous les outils en main pour comparer les solutions de KP-II et KP-BBM-II. On étudiera la différence entre $u$ et $v$, solution du problème de Cauchy (2.5)-(2.7) pour laquelle le temps maximum de comparaison est de l'ordre d'une constante, on en déduira alors des résultats sur $\eta$ et $\zeta$, solution du problème de Cauchy (2.1)-(2.3).

\subsection{Comparaison des solutions}

Théorème 2.9. Soient $m \geq 0$, et $f \in X^{m+5}\left(\mathbb{R}^{2}\right)$. Soit $\alpha>0$ et soient $\eta$ et $\zeta$ l'unique solution du problème de Cauchy (2.1)-(2.2)-(2.3) dans $\mathcal{C}\left(\left[-\alpha^{-1} T_{0}, \alpha^{-1} T_{0}\right] ; X^{m+5}\left(\mathbb{R}^{2}\right)\right)$, avec $T_{0}:=\frac{1}{C_{m+5}|f|_{m+5}}$. Alors, il existe $\alpha_{0}>0$, et pour tous entiers naturels $0 \leq i+j \leq m$, une constante $M_{i, j}>0$, dépendant uniquement de $|f|_{m+5}$, telle que si $0<\alpha \leq \alpha_{0}$, et pour $|t| \leq \alpha^{-1} T_{0}$, on ait

$$
\left\|\partial_{x}^{i} \partial_{y}^{j} \eta(., ., t)-\partial_{x}^{i} \partial_{y}^{j} \zeta(., ., t)\right\| \leq M_{i, j} \alpha^{3 / 2}|t|^{1 / 2}
$$

Remarque 2.10. Notons que l'existence et l'unicité des solutions $\eta$ et $\zeta$ sont assurées par la section précédente.

Démonstration. On va montrer ce théorème par récurrence sur le nombre total de dérivées, autrement dit sur $i+j$. On suppose que le temps est positif, la preuve s'adaptera de la même manière dans le cas où le temps est négatif.

On effectue tout d'abord le changement de fonctions

$$
u(x, y, t)=\eta\left(x+\alpha^{-1} t, y, \alpha^{-1} t\right), v(x, y, t)=\zeta\left(x+\alpha^{-1} t, y, \alpha^{-1} t\right),
$$

ce qui nous permet de retrouver exactement les équations (2.5), (2.6) et (2.7). Posons $w=v-u$, alors $w$ satisfait le problème de Cauchy

$$
\begin{aligned}
w_{t}+w w_{x}+w_{x x x}-\alpha w_{x x t}+\partial_{x}^{-1} w_{y y} & =\alpha u_{x x t}-(u w)_{x} \\
w(x, y, 0) & =0 .
\end{aligned}
$$


Pour $i+j=0$, c'est-à-dire pour $i=j=0$, on multiplie l'équation (2.14) par $w$ et intègre en espace

$$
\int_{\mathbb{R}^{2}}\left[w w_{t}+w^{2} w_{x}+w w_{x x x}-\alpha w w_{x x t}+w \partial_{x}^{-1} w_{y y}\right] \mathrm{d} x \mathrm{~d} y=\int_{\mathbb{R}^{2}}\left[\alpha w u_{x x t}-w(u w)_{x}\right] \mathrm{d} x \mathrm{~d} y .
$$

Simplifions l'intégrale de gauche : comme $w \in H^{m+5}$ et $\partial_{x}^{-1} w \in H^{m+5}$, en particulier, $\partial_{x}^{i} \partial_{y}^{j} w(x, y)$ et $\partial_{x}^{i-1} \partial_{y}^{j} w(x, y)$, pour $0 \leq i+j \leq m+5$, tendent vers zéro à l'infini. On a alors

$$
\int_{\mathbb{R}^{2}} w^{2} w_{x}=\int_{-\infty}^{+\infty}\left[\frac{w^{3}}{3}\right]_{-\infty}^{+\infty} \mathrm{d} y=0
$$

et une intégration par parties donne

$$
\begin{aligned}
\int_{\mathbb{R}^{2}}\left[w w_{x x x}+w \partial_{x}^{-1} w_{y y}\right] \mathrm{d} x \mathrm{~d} y & =-\int_{\mathbb{R}^{2}}\left[w_{x} w_{x x}+w_{y} \partial_{x}^{-1} w_{y}\right] \mathrm{d} x \mathrm{~d} y \\
& =-\int_{-\infty}^{+\infty}\left[\frac{w_{x}^{2}}{2}+\frac{\left(\partial_{x}^{-1} w_{y}\right)^{2}}{2}\right]_{-\infty}^{+\infty} \mathrm{d} y=0 .
\end{aligned}
$$

Il reste alors, après une dernière intégration par parties, et l'utilisation du théorème de dérivation de Lebesgue,

$$
\begin{aligned}
\int_{\mathbb{R}^{2}}\left[w w_{t}+w^{2} w_{x}+w w_{x x x}-\alpha w w_{x x t}+w \partial_{x}^{-1} w_{y y}\right] \mathrm{d} x \mathrm{~d} y & =\int_{\mathbb{R}^{2}}\left[w w_{t}+\alpha w_{x} w_{x t}\right] \mathrm{d} x \mathrm{~d} y \\
& =\frac{1}{2} \frac{\mathrm{d}}{\mathrm{d} t} \int_{\mathbb{R}^{2}}\left[w^{2}+\alpha w_{x}^{2}\right] \mathrm{d} x \mathrm{~d} y
\end{aligned}
$$

Simplifions maintenant l'intégrale de droite : par intégration par parties, on a

$$
\begin{aligned}
\int_{\mathbb{R}^{2}}\left[\alpha w u_{x x t}-w(u w)_{x}\right] \mathrm{d} x \mathrm{~d} y & =\int_{\mathbb{R}^{2}}\left[\alpha w u_{x x t}+w_{x} u w\right] \mathrm{d} x \mathrm{~d} y \\
& =\int_{\mathbb{R}^{2}}\left[\alpha w u_{x x t}+u\left(\frac{w^{2}}{2}\right)_{x}\right] \mathrm{d} x \mathrm{~d} y
\end{aligned}
$$

et une nouvelle intégration par parties donne

$$
\int_{\mathbb{R}^{2}}\left[\alpha w u_{x x t}-w(u w)_{x}\right] \mathrm{d} x \mathrm{~d} y=\int_{\mathbb{R}^{2}}\left[\alpha w u_{x x t}-\frac{w^{2}}{2} u_{x}\right] \mathrm{d} x \mathrm{~d} y .
$$

Finalement, on a

$$
\frac{\mathrm{d}}{\mathrm{d} t} \int_{\mathbb{R}^{2}}\left[w^{2}+\alpha w_{x}^{2}\right] \mathrm{d} x \mathrm{~d} y=\int_{\mathbb{R}^{2}}\left[2 \alpha w u_{x x t}-w^{2} u_{x}\right] \mathrm{d} x \mathrm{~d} y,
$$

ou encore, comme $w(x, y, 0)=0$,

$$
\int_{\mathbb{R}^{2}}\left[w^{2}+\alpha w_{x}^{2}\right] \mathrm{d} x \mathrm{~d} y=\int_{0}^{t} \int_{\mathbb{R}^{2}}\left[2 \alpha w u_{x x \tau}-w^{2} u_{x}\right] \mathrm{d} x \mathrm{~d} y \mathrm{~d} \tau .
$$

On en déduit, en utilisant l'inégalité de Cauchy-Schwarz,

$$
\begin{aligned}
\|w(t)\|^{2} & \leq 2 \alpha \int_{0}^{t}\|w(\tau)\|\left\|u_{x x \tau}(\tau)\right\| \mathrm{d} \tau+\int_{0}^{t}\|w(\tau)\|^{2}\left\|u_{x}(\tau)\right\|_{\infty} \mathrm{d} \tau \\
& \leq 2 \alpha c_{2} \int_{0}^{t}\|w(\tau)\| \mathrm{d} \tau+c_{1} \int_{0}^{t}\|w(\tau)\|^{2} \mathrm{~d} \tau
\end{aligned}
$$


pour $t \in\left[0, T_{0}\right]$, où les constantes $c_{1}$ et $c_{2}$ sont déterminées de telle sorte qu'elles majorent respectivement $\left\|u_{x}(\tau)\right\|_{\infty}$ et $\left\|u_{x x \tau}(\tau)\right\|$, pour $\tau \in[0, t]$, indépendamment du temps et de $\alpha$. En effet d'après la Proposition 2.2, on a pour $t \in\left[0, T_{0}\right]$

D'où, via l'inégalité (2.13),

$$
|| u(t)||_{m+5} \leq c_{m+5}|f|_{m+5} .
$$

$$
\sup _{\tau \in\left[0, T_{0}\right]}\left\|u_{x}(\tau)\right\|_{\infty} \leq 2 \sup _{\tau \in\left[0, T_{0}\right]}\left(\left\|u_{x}\right\|\left\|u_{x x}\right\|\left\|u_{x y}\right\|\left\|u_{x x y}\right\|(\tau)\right)^{1 / 4} \leq c_{1},
$$

et

$$
\begin{aligned}
\sup _{\tau \in\left[0, T_{0}\right]}\left\|u_{x x \tau}(\tau)\right\| & =\sup _{\tau \in\left[0, T_{0}\right]}\left\|\partial_{x}^{2}\left(u_{x x x}+u u_{x}+\partial_{x}^{-1} u_{y y}\right)(\tau)\right\| \\
& \leq \sup _{\tau \in\left[0, T_{0}\right]}\left(\left\|u_{x x x x x}(\tau)\right\|+3\left\|u_{x}(\tau)\right\|\left\|u_{x x}(\tau)\right\|+\|u(\tau)\|\left\|u_{x x x}(\tau)\right\|+\left\|u_{x y y}(\tau)\right\|\right) \\
& \leq c_{2} .
\end{aligned}
$$

Comme, pour $a$ et $b$ réels, $2 a b \leq a^{2}+b^{2}$, on a

$$
\|w(t)\|^{2} \leq\left(\alpha c_{2}\right)^{2} t+\left(1+c_{1}\right) \int_{0}^{t}\|w(\tau)\|^{2} \mathrm{~d} \tau
$$

On utilise le Lemme 2.8 de type Gronwall avec $f(t)=\|w(t)\|^{2}, g(t)=\left(\alpha c_{2}\right)^{2} t$ et $c=\left(1+c_{1}\right)$, on trouve

$$
\begin{aligned}
\|w(t)\|^{2} & \leq\left(\alpha c_{2}\right)^{2} t+\left(1+c_{1}\right) \mathrm{e}^{\left(1+c_{1}\right) t} \int_{0}^{t}\left(\alpha c_{2}\right)^{2} \tau \mathrm{e}^{-\left(1+c_{1}\right) \tau} \mathrm{d} \tau \\
& =\left(\alpha c_{2}\right)^{2} \frac{\left(\mathrm{e}^{\left(1+c_{1}\right) t}-1\right)}{1+c_{1}} .
\end{aligned}
$$

Or,

$$
\frac{\left(\mathrm{e}^{\left(1+c_{1}\right) t}-1\right)}{1+c_{1}}=\frac{1+\left(1+c_{1}\right) t+\sum_{n=2}^{+\infty}\left(1+c_{1}\right)^{n} t^{n}-1}{1+c_{1}}=t\left(1+\sum_{n=1}^{+\infty}\left(1+c_{1}\right)^{n} t^{n}\right),
$$

d'où, pour $0 \leq t \leq T_{0}=\frac{1}{C_{m+5}|f|_{m+5}}$,

$$
\frac{\left(\mathrm{e}^{\left(1+c_{1}\right) t}-1\right)}{1+c_{1}} \leq t \mathrm{e}^{\frac{\left(1+c_{1}\right)|f|_{m+5}}{C_{m+5}}}
$$

Finalement, on a pour $0 \leq t \leq T_{0}$

$$
\|w(t)\| \leq c_{2} \mathrm{e}^{\frac{\left(1+c_{1}\right)|f|_{m+5}}{2 C_{m}+5}} \alpha t^{1 / 2}=M_{0} \alpha t^{1 / 2},
$$

avec $M_{0}$ qui ne dépend que de $|f|_{m+5}$.

On suppose que le résultat est vrai jusqu'au rang $m-1$, plus précisément pour $0 \leq i+j \leq m-1$, et $0 \leq t \leq T_{0}$, il existe une constante $M_{i, j}>0$ telle que

$$
\left\|\partial_{x}^{i} \partial_{y}^{j} w(t)\right\| \leq M_{i, j} \alpha t^{1 / 2}
$$

et montrons qu'il reste vrai au rang $m$. 
Il s'agira d'obtenir une inégalité du type (2.15), la seule différence sera que cette fois-ci, le terme cubique en $w$ ne s'élimine pas par intégration. On utilisera le fait que $w$ est bornée en norme $L^{\infty}\left(\mathbb{R}^{2}\right)$ et l'hypothèse de récurrence.

On note $\partial_{x}^{2 i} \partial_{y}^{2 j} w$ la dérivée d'ordre $2 i$ dans la direction $x$ et $2 j$ dans la direction $y$, de telle sorte que $i+j=m$. On multiplie alors l'équation (2.14) par $\partial_{x}^{2 i} \partial_{y}^{2 j} w$ et intègre en espace

$$
\int_{\mathbb{R}^{2}}\left[w_{t}+w w_{x}+w_{x x x}-\alpha w_{x x t}+\partial_{x}^{-1} w_{y y}\right] \partial_{x}^{2 i} \partial_{y}^{2 j} w \mathrm{~d} x \mathrm{~d} y=\int_{\mathbb{R}^{2}}\left[\alpha u_{x x t}-(u w)_{x}\right] \partial_{x}^{2 i} \partial_{y}^{2 j} w \mathrm{~d} x \mathrm{~d} y .
$$

Simplifions l'intégrale de gauche : en effectuant $i$ intégrations par parties dans la direction $x$ et $j$ intégrations par parties en $y$, on trouve

$$
\begin{aligned}
\int_{\mathbb{R}^{2}}\left[\partial_{x}^{2 i} \partial_{y}^{2 j} w w_{x x x}+\partial_{x}^{2 i} \partial_{y}^{2 j} w \partial_{x}^{-1} w_{y y}\right] \mathrm{d} x \mathrm{~d} y & =(-1)^{i+j} \int_{\mathbb{R}^{2}}\left[\partial_{x}^{i+1} \partial_{y}^{j} w \partial_{x}^{i+2} \partial_{y}^{j} w+\partial_{x}^{i} \partial_{y}^{j+1} w \partial_{x}^{i-1} \partial_{y}^{j+1} w\right] \mathrm{d} x \mathrm{~d} y \\
& =(-1)^{i+j} \int_{-\infty}^{+\infty}\left[\frac{\left(\partial_{x}^{i+1} \partial_{y}^{j} w\right)^{2}}{2}+\frac{\left(\partial_{x}^{i-1} \partial_{y}^{j+1} w\right)^{2}}{2}\right]_{-\infty}^{+\infty} \mathrm{d} y \\
& =0, \\
\int_{\mathbb{R}^{2}}\left[\partial_{x}^{2 i} \partial_{y}^{2 j} w w w_{x}\right] \mathrm{d} x \mathrm{~d} y & =(-1)^{i+j} \int_{\mathbb{R}^{2}}\left[\partial_{x}^{i} \partial_{y}^{j} w \frac{\partial_{x}^{i+1} \partial_{y}^{j}\left(w^{2}\right)}{2}\right] \mathrm{d} x \mathrm{~d} y
\end{aligned}
$$

et

$$
\int_{\mathbb{R}^{2}}\left[\partial_{x}^{2 i} \partial_{y}^{2 j} w w_{t}-\alpha \partial_{x}^{2 i} \partial_{y}^{2 j} w w_{x x t}\right] \mathrm{d} x \mathrm{~d} y=(-1)^{i+j} \int_{\mathbb{R}^{2}}\left[\partial_{x}^{i} \partial_{y}^{j} w \partial_{x}^{i} \partial_{y}^{j} w_{t}+\alpha \partial_{x}^{i+1} \partial_{y}^{j} w \partial_{x}^{i+1} \partial_{y}^{j} w_{t}\right] \mathrm{d} x \mathrm{~d} y .
$$

Le théorème de dérivation de Lebesgue implique que

$$
\int_{\mathbb{R}^{2}}\left[\partial_{x}^{2 i} \partial_{y}^{2 j} w w_{t}-\alpha \partial_{x}^{2 i} \partial_{y}^{2 j} w w_{x x t}\right] \mathrm{d} x \mathrm{~d} y=\frac{(-1)^{i+j}}{2} \frac{\mathrm{d}}{\mathrm{d} t} \int_{\mathbb{R}^{2}}\left[\left(\partial_{x}^{i} \partial_{y}^{j} w\right)^{2}+\alpha\left(\partial_{x}^{i+1} \partial_{y}^{j} w\right)^{2}\right] \mathrm{d} x \mathrm{~d} y .
$$

Simplifions désormais l'intégrale de droite : par intégration par parties, on a

$$
\int_{\mathbb{R}^{2}}\left[\alpha \partial_{x}^{2 i} \partial_{y}^{2 j} w u_{x x t}-\partial_{x}^{2 i} \partial_{y}^{2 j} w(u w)_{x}\right] \mathrm{d} x \mathrm{~d} y=(-1)^{i+j} \int_{\mathbb{R}^{2}}\left[\alpha \partial_{x}^{i} \partial_{y}^{j} w \partial_{x}^{i+2} \partial_{y}^{j} u_{t}-\partial_{x}^{i} \partial_{y}^{j} w \partial_{x}^{i+1} \partial_{y}^{j}(u w)\right] \mathrm{d} x \mathrm{~d} y .
$$

Finalement, comme $w(x, y, 0)=0$, on obtient

$$
\int_{\mathbb{R}^{2}}\left(\partial_{x}^{i} \partial_{y}^{j} w\right)^{2}+\alpha\left(\partial_{x}^{i+1} \partial_{y}^{j} w\right)^{2} \mathrm{~d} x \mathrm{~d} y=2 \int_{0}^{t} \int_{\mathbb{R}^{2}} \partial_{x}^{i} \partial_{y}^{j} w\left(\alpha \partial_{x}^{i+2} \partial_{y}^{j} u_{\tau}-\partial_{x}^{i+1} \partial_{y}^{j}\left(u w+\frac{w^{2}}{2}\right)\right) \mathrm{d} x \mathrm{~d} y \mathrm{~d} \tau .
$$

On applique la formule de Leibniz pour obtenir

$$
\begin{aligned}
\int_{\mathbb{R}^{2}}\left(\partial_{x}^{i} \partial_{y}^{j} w\right)^{2}+\alpha\left(\partial_{x}^{i+1} \partial_{y}^{j} w\right)^{2} \mathrm{~d} x \mathrm{~d} y & =2 \alpha \int_{0}^{t} \int_{\mathbb{R}^{2}} \partial_{x}^{i} \partial_{y}^{j} w \partial_{x}^{i+2} \partial_{y}^{j} u_{\tau} \mathrm{d} x \mathrm{~d} y \mathrm{~d} \tau \\
& -\int_{0}^{t} \int_{\mathbb{R}^{2}} \sum_{r=0}^{i+1} \sum_{s=0}^{j}\left(\begin{array}{c}
i+1 \\
r
\end{array}\right)\left(\begin{array}{c}
j \\
s
\end{array}\right)\left(\partial_{x}^{i+1-r} \partial_{y}^{j-s} w \partial_{x}^{r} \partial_{y}^{s} w\right) \partial_{x}^{i} \partial_{y}^{j} w \mathrm{~d} x \mathrm{~d} y \mathrm{~d} \tau \\
& -2 \int_{0}^{t} \int_{\mathbb{R}^{2}}^{i+1} \sum_{r=0}^{j} \sum_{s=0}^{j}\left(\begin{array}{c}
i+1 \\
r
\end{array}\right)\left(\begin{array}{c}
j \\
s
\end{array}\right)\left(\partial_{x}^{i+1-r} \partial_{y}^{j-s} w \partial_{x}^{r} \partial_{y}^{s} u\right) \partial_{x}^{i} \partial_{y}^{j} w \mathrm{~d} x \mathrm{~d} y \mathrm{~d} \tau,
\end{aligned}
$$


puis on sépare les dérivées d'ordre $m+1$,

$$
\begin{aligned}
\int_{\mathbb{R}^{2}}\left(\partial_{x}^{i} \partial_{y}^{j} w\right)^{2}+\alpha\left(\partial_{x}^{i+1} \partial_{y}^{j} w\right)^{2} \mathrm{~d} x \mathrm{~d} y & =2 \alpha \int_{0}^{t} \int_{\mathbb{R}^{2}} \partial_{x}^{i} \partial_{y}^{j} w \partial_{x}^{i+2} \partial_{y}^{j} u_{\tau} \mathrm{d} x \mathrm{~d} y \mathrm{~d} \tau \\
& -2 \int_{0}^{t} \int_{\mathbb{R}^{2}}\left[w \partial_{x}^{i+1} \partial_{y}^{j} w \partial_{x}^{i} \partial_{y}^{j} w+u \partial_{x}^{i+1} \partial_{y}^{j} w \partial_{x}^{i} \partial_{y}^{j} w\right] \mathrm{d} x \mathrm{~d} y \mathrm{~d} \tau \\
& -\int_{0}^{t} \int_{\mathbb{R}^{2}} \sum_{r=1}^{i+1} \sum_{s=1}^{j}\left(\begin{array}{c}
i+1 \\
r
\end{array}\right)\left(\begin{array}{c}
j \\
s
\end{array}\right) \partial_{x}^{i+1-r} \partial_{y}^{j-s} w \partial_{x}^{r} \partial_{y}^{s} w \partial_{x}^{i} \partial_{y}^{j} w \mathrm{~d} x \mathrm{~d} y \mathrm{~d} \tau \\
& -2 \int_{0}^{t} \int_{\mathbb{R}^{2}} \sum_{r=1}^{i+1} \sum_{s=1}^{j}\left(\begin{array}{c}
i+1 \\
r
\end{array}\right)\left(\begin{array}{c}
j \\
s
\end{array}\right) \partial_{x}^{i+1-r} \partial_{y}^{j-s} w \partial_{x}^{r} \partial_{y}^{s} u \partial_{x}^{i} \partial_{y}^{j} w \mathrm{~d} x \mathrm{~d} y \mathrm{~d} \tau
\end{aligned}
$$

La première intégrale du membre de droite de (2.16) est majorée, d'après l'inégalité de Cauchy-Schwarz, sur $\left[0, T_{0}\right]$, par

$$
\begin{aligned}
\left|2 \alpha \int_{0}^{t} \int_{\mathbb{R}^{2}} \partial_{x}^{i} \partial_{y}^{j} w(\tau) \partial_{x}^{i+2} \partial_{y}^{j} u_{\tau}(\tau) \mathrm{d} x \mathrm{~d} y \mathrm{~d} \tau\right| & \leq 2 \alpha \int_{0}^{t}\left\|\partial_{x}^{i} \partial_{y}^{j} w(\tau)\right\|\left\|\partial_{x}^{i+2} \partial_{y}^{j} u_{\tau}(\tau)\right\| \mathrm{d} \tau \\
& \leq \alpha c \int_{0}^{t}\left\|w_{i, j}(\tau)\right\| \mathrm{d} \tau
\end{aligned}
$$

car d'après la Proposition 2.2

$$
\begin{aligned}
\sup _{\tau \in\left[0, T_{0}\right]}\left\|\partial_{x}^{i+2} \partial_{y}^{j} u_{\tau}(\tau)\right\| & =\sup _{\tau \in\left[0, T_{0}\right]}\left\|\partial_{x}^{i+2} \partial_{y}^{j}\left(u_{x x x}+u u_{x}+\partial_{x}^{-1} u_{y y}\right)(\tau)\right\| \\
& \leq c
\end{aligned}
$$

Par abus, on continuera de noter $c$ les différentes constantes intervenant dans les majorations. La seconde intégrale de (2.16) donne, après une intégration par parties dans la direction $x$,

$$
\begin{aligned}
\left|\int_{0}^{t} \int_{\mathbb{R}^{2}}\left(w_{x}(\tau)+u_{x}(\tau)\right)\left(\partial_{x}^{i} \partial_{y}^{j} w\right)^{2}(\tau) \mathrm{d} x \mathrm{~d} y \mathrm{~d} \tau\right| & \leq \int_{0}^{t}\left(\left\|w_{x}(\tau)\right\|_{\infty}+\left\|u_{x}(\tau)\right\|_{\infty}\right)\left\|\partial_{x}^{i} \partial_{y}^{j} w(\tau)\right\|^{2} \mathrm{~d} \tau \\
& \leq \int_{0}^{t}\left(\left\|v_{x}(\tau)\right\|_{\infty}+2\left\|u_{x}(\tau)\right\|_{\infty}\right)\left\|\partial_{x}^{i} \partial_{y}^{j} w(\tau)\right\|^{2} \mathrm{~d} \tau
\end{aligned}
$$

et d'après l'inégalité (2.13) et les propositions 2.2 et 2.3 , on a pour $t \in\left[0, T_{0}\right]$

$$
\left|\int_{0}^{t} \int_{\mathbb{R}^{2}}\left(w_{x}(\tau)+u_{x}(\tau)\right)\left(\partial_{x}^{i} \partial_{y}^{j} w\right)^{2} \mathrm{~d} x \mathrm{~d} y \mathrm{~d} \tau\right| \leq c \int_{0}^{t}\left\|\partial_{x}^{i} \partial_{y}^{j} w(\tau)\right\|^{2} \mathrm{~d} \tau
$$

La troisième intégrale de (2.16) est majorée d'après l'inégalité de Cauchy-Schwarz,

$$
\begin{aligned}
\left|\int_{0}^{t} \int_{\mathbb{R}^{2}} \sum_{r=1}^{i+1} \sum_{s=1}^{j}\left(\begin{array}{c}
i+1 \\
r
\end{array}\right)\left(\begin{array}{c}
j \\
s
\end{array}\right) \partial_{x}^{i+1-r} \partial_{y}^{j-s} w(\tau) \partial_{x}^{r} \partial_{y}^{s} w(\tau) \partial_{x}^{i} \partial_{y}^{j} w(\tau) \mathrm{d} x \mathrm{~d} y \mathrm{~d} \tau\right| \leq \\
\qquad \sum_{r=1}^{i+1} \sum_{s=1}^{j}\left(\begin{array}{c}
i+1 \\
r
\end{array}\right)\left(\begin{array}{c}
j \\
s
\end{array}\right) \int_{0}^{t}\left\|\partial_{x}^{r} \partial_{y}^{s} w(\tau)\right\|_{\infty}\left\|\partial_{x}^{i} \partial_{y}^{j} w(\tau)\right\|\left\|\partial_{x}^{i+1-r} \partial_{y}^{j-s} w(\tau)\right\| \mathrm{d} \tau .
\end{aligned}
$$


Or, pour $1 \leq r \leq i+1$ et $1 \leq s \leq j$, on a $2 \leq r+s \leq m+1$ et $0 \leq(i+1-r)+(j-s) \leq m-1$. D'après les Propositions 2.2 et 2.3 , pour tout $\tau \in\left[0, T_{0}\right]$, on a, en utilisant l'inégalité (2.13), pour $1 \leq r \leq i+1$ et $1 \leq s \leq j$,

$$
\begin{aligned}
\left\|\partial_{x}^{r} \partial_{y}^{s} w(\tau)\right\|_{\infty} & \leq\left\|\partial_{x}^{r} \partial_{y}^{s} v(\tau)\right\|_{\infty}+\left\|\partial_{x}^{r} \partial_{y}^{s} u(\tau)\right\|_{\infty} \\
& \leq c
\end{aligned}
$$

et l'hypothèse de récurrence implique que pour $\tau \in\left[0, T_{0}\right]$

$$
\begin{gathered}
\sum_{r=1}^{i+1} \sum_{s=1}^{j}\left(\begin{array}{c}
i+1 \\
r
\end{array}\right)\left(\begin{array}{c}
j \\
s
\end{array}\right) \int_{0}^{t}\left\|\partial_{x}^{r} \partial_{y}^{s} w(\tau)\right\|_{\infty}\left\|\partial_{x}^{i} \partial_{y}^{j} w(\tau)\right\|\left\|\partial_{x}^{i+1-r} \partial_{y}^{j-s} w(\tau)\right\| \mathrm{d} \tau \leq \\
\alpha c \sum_{r=1}^{i+1} \sum_{s=1}^{j}\left(\begin{array}{c}
i+1 \\
r
\end{array}\right)\left(\begin{array}{c}
j \\
s
\end{array}\right) M_{i+1-r, j-s} \int_{0}^{t} \tau^{1 / 2}\left\|\partial_{x}^{i} \partial_{y}^{j} w(\tau)\right\| \mathrm{d} \tau,
\end{gathered}
$$

et comme $0 \leq \tau \leq T_{0}$,

$$
\begin{gathered}
\sum_{r=1}^{i+1} \sum_{s=1}^{j}\left(\begin{array}{c}
i+1 \\
r
\end{array}\right)\left(\begin{array}{c}
j \\
s
\end{array}\right) \int_{0}^{t}\left\|\partial_{x}^{r} \partial_{y}^{s} w(\tau)\right\|_{\infty}\left\|\partial_{x}^{i} \partial_{y}^{j} w(\tau)\right\|\left\|\partial_{x}^{i+1-r} \partial_{y}^{j-s} w(\tau)\right\| \mathrm{d} \tau \leq \\
\alpha c \sum_{r=1}^{i+1} \sum_{s=1}^{j}\left(\begin{array}{c}
i+1 \\
r
\end{array}\right)\left(\begin{array}{c}
j \\
s
\end{array}\right) M_{i+1-r, j-s} \int_{0}^{t}\left\|\partial_{x}^{i} \partial_{y}^{j} w(\tau)\right\| \mathrm{d} \tau .
\end{gathered}
$$

Finalement, on a trouvé que la troisième intégrale est majorée par

$$
\left|\int_{0}^{t} \int_{\mathbb{R}^{2}} \sum_{r=1}^{i+1} \sum_{s=1}^{j}\left(\begin{array}{c}
i+1 \\
r
\end{array}\right)\left(\begin{array}{c}
j \\
s
\end{array}\right) \partial_{x}^{i+1-r} \partial_{y}^{j-s} w(\tau) \partial_{x}^{r} \partial_{y}^{s} w(\tau) \partial_{x}^{i} \partial_{y}^{j} w(\tau) \mathrm{d} x \mathrm{~d} y \mathrm{~d} \tau\right| \leq \alpha c \int_{0}^{t}\left\|\partial_{x}^{i} \partial_{y}^{j} w(\tau)\right\| \mathrm{d} \tau .
$$

La dernière intégrale de (2.16) est majorée par

$$
\begin{aligned}
\left|\int_{0}^{t} \int_{\mathbb{R}^{2}} \sum_{r=1}^{i+1} \sum_{s=1}^{j}\left(\begin{array}{c}
i+1 \\
r
\end{array}\right)\left(\begin{array}{c}
j \\
s
\end{array}\right) \partial_{x}^{i+1-r} \partial_{y}^{j-s} w(\tau) \partial_{x}^{r} \partial_{y}^{s} u(\tau) \partial_{x}^{i} \partial_{y}^{j} w(\tau) \mathrm{d} x \mathrm{~d} y \mathrm{~d} \tau\right| \leq \\
\qquad \sum_{r=1}^{i+1} \sum_{s=1}^{j}\left(\begin{array}{c}
i+1 \\
r
\end{array}\right)\left(\begin{array}{c}
j \\
s
\end{array}\right) \int_{0}^{t}\left\|\partial_{x}^{r} \partial_{y}^{s} u(\tau)\right\|_{\infty}\left\|\partial_{x}^{i} \partial_{y}^{j} w(\tau)\right\|\left\|\partial_{x}^{i+1-r} \partial_{y}^{j-s} w(\tau)\right\| \mathrm{d} \tau .
\end{aligned}
$$

D'après l'inégalité (2.13) et la Proposition 2.2, pour tout $\tau \in\left[0, T_{0}\right]$, on a

$$
\left\|\partial_{x}^{r} \partial_{y}^{s} u(\tau)\right\|_{\infty} \leq c
$$

et l'hypothèse de récurrence implique que pour $\tau \in\left[0, T_{0}\right]$

$$
\begin{gathered}
\sum_{r=1}^{i+1} \sum_{s=1}^{j}\left(\begin{array}{c}
i+1 \\
r
\end{array}\right)\left(\begin{array}{c}
j \\
s
\end{array}\right) \int_{0}^{t}\left\|\partial_{x}^{r} \partial_{y}^{s} u(\tau)\right\|_{\infty}\left\|\partial_{x}^{i} \partial_{y}^{j} w(\tau)\right\|\left\|\partial_{x}^{i+1-r} \partial_{y}^{j-s} w(\tau)\right\| \mathrm{d} \tau \leq \\
\alpha c \sum_{r=1}^{i+1} \sum_{s=1}^{j}\left(\begin{array}{c}
i+1 \\
r
\end{array}\right)\left(\begin{array}{c}
j \\
s
\end{array}\right) M_{i+1-r, j-s} \int_{0}^{t}\left\|\partial_{x}^{i} \partial_{y}^{j} w(\tau)\right\| \mathrm{d} \tau .
\end{gathered}
$$


Finalement, on a trouvé que la dernière intégrale est majorée par

$$
\left|2 \int_{0}^{t} \int_{\mathbb{R}^{2}} \sum_{r=1}^{i+1} \sum_{s=1}^{j}\left(\begin{array}{c}
i+1 \\
r
\end{array}\right)\left(\begin{array}{c}
j \\
s
\end{array}\right) \partial_{x}^{i+1-r} \partial_{y}^{j-s} w(\tau) \partial_{x}^{r} \partial_{y}^{s} u(\tau) \partial_{x}^{i} \partial_{y}^{j} w(\tau) \mathrm{d} x \mathrm{~d} y \mathrm{~d} \tau\right| \leq \alpha c \int_{0}^{t}\left\|\partial_{x}^{i} \partial_{y}^{j} w(\tau)\right\| \mathrm{d} \tau
$$

En résumé, on a sur l'intervalle de temps $\left[0, T_{0}\right]$,

$$
\left\|\partial_{x}^{i} \partial_{y}^{j} w(t)\right\|^{2} \leq \alpha c_{1} \int_{0}^{t}\left\|\partial_{x}^{i} \partial_{y}^{j} w(\tau)\right\| \mathrm{d} \tau+c_{2} \int_{0}^{t}\left\|\partial_{x}^{i} \partial_{y}^{j} w(\tau)\right\|^{2} \mathrm{~d} \tau
$$

On conclut de la même manière qu'au premier rang à l'aide du Lemme 2.8. Il suffit alors de remarquer que

$$
\left\|\partial_{x}^{i} \partial_{y}^{j} \eta(., ., t)-\partial_{x}^{i} \partial_{y}^{j} \zeta(., ., t)\right\|=\left\|\partial_{x}^{i} \partial_{y}^{j} w(\alpha t)\right\| \leq M_{i, j} \alpha^{3 / 2} t^{1 / 2}
$$

Corollaire 2.11. Soient $m \geq 2$, et $f \in X^{m+5}\left(\mathbb{R}^{2}\right)$. Soit $\alpha>0$ et soient $\eta$ et $\zeta$ l'unique solution du problème de Cauchy (2.1)-(2.2)-(2.3) dans $\mathcal{C}\left(\left[-\alpha^{-1} T_{0}, \alpha^{-1} T_{0}\right] ; X^{m+5}\left(\mathbb{R}^{2}\right)\right)$, avec $T_{0}:=\frac{1}{C_{m+5}|f|_{m+5}}$. Alors, il existe $\alpha_{0}>0$, et pour tous entiers naturels $0 \leq i+j \leq m-2$, une constante $N_{i, j}>0$, dépendant uniquement de $|f|_{m+5}$, telle que si $0<\alpha \leq \alpha_{0}$, et pour $|t| \leq \alpha^{-1} T_{0}$, on ait

$$
\left\|\partial_{x}^{i} \partial_{y}^{j} \eta(., ., t)-\partial_{x}^{i} \partial_{y}^{j} \zeta(., ., t)\right\|_{L^{\infty}\left(\mathbb{R}^{2}\right)} \leq N_{i, j} \alpha^{3 / 2}|t|^{1 / 2}
$$

Démonstration. L’inégalité (2.13) implique

$$
\left\|\partial_{x}^{i} \partial_{y}^{j}(\eta-\zeta)\right\|_{L^{\infty}\left(\mathbb{R}^{2}\right)} \leq 2\left(\left\|\partial_{x}^{i} \partial_{y}^{j}(\eta-\zeta)\right\|\left\|\partial_{x}^{i+1} \partial_{y}^{j}(\eta-\zeta)\right\|\left\|\partial_{x}^{i} \partial_{y}^{j+1}(\eta-\zeta)\right\|\left\|\partial_{x}^{i+1} \partial_{y}^{j+1}(\eta-\zeta)\right\|\right)^{1 / 4} .
$$

Le théorème précédent s'applique et donne, pour $|t| \leq \alpha^{-1} T_{0}$,

$$
\left\|\partial_{x}^{i} \partial_{y}^{j}(\eta-\zeta)(t)\right\|_{L^{\infty}\left(\mathbb{R}^{2}\right)} \leq 2\left(M_{i, j} \alpha^{3 / 2}|t|^{1 / 2} M_{i+1, j} \alpha^{3 / 2}|t|^{1 / 2} M_{i, j+1} \alpha^{3 / 2}|t|^{1 / 2} M_{i+1, j+1} \alpha^{3 / 2}|t|^{1 / 2}\right)^{1 / 4}
$$

Il suffit de prendre $N_{i, j}=2\left(M_{i, j} M_{i+1, j} M_{i, j+1} M_{i+1, j+1}\right)^{1 / 4}$.

Remarque 2.12. On a remarqué que les équations de KP-II et KP-BBM-II sont des corrections au premier ordre de l'équation $\eta_{t}+\eta_{x}=O(\alpha)$. On peux alors obtenir une troisième équation de type KP [16]

$$
\xi_{t}+\xi_{x}+\alpha \xi \xi_{x}+\alpha \xi_{x t t}+\partial_{x}^{-1} \xi_{y y}=0
$$

Il est tentant de vouloir comparer les solutions de cette dernière équation avec celles de KP-II et KP-BBM-II. Bona et Chen [4] montre en dimension 1 un résultat similaire au Corollaire 2.11 pour un temps maximum de comparaison d'ordre $\alpha^{-1 / 2}$. Dans le cas de la dimension 2, la transformée Fourier en espace de l'équation (2.17) donne une équation différentielle ordinaire du second ordre, pour laquelle les solutions de l'équation caractéristique peuvent être doubles, ce qui n'apparaissait pas en dimension 1. Dans le cas de racines simples, on retrouve le résultat de Bona et Chen [4], mais en cas de racines doubles, le temps de comparaison devient d'ordre $\alpha$, ce qui est bien trop court pour comparer les solutions. Il serait intéressant de voir s'il est possible de décrire les modes pour lesquelles les racines doubles apparaissent. 


\subsection{Le cas périodique}

Dans l'article [6] de Bona et al. (voir le Thm. 0.1 de l'introduction), on considère les équations de KdV et BBM sous la forme

$$
\begin{aligned}
\eta_{t}+\eta_{x}+\alpha \eta \eta_{x}+\alpha \eta_{x x x} & =0 \\
\zeta_{t}+\zeta_{x}+\alpha \zeta \zeta_{x}-\alpha \zeta_{x x t} & =0 \\
\eta(x, 0)=\zeta(x, 0) & =f(x)
\end{aligned}
$$

Le passage de ces équations à celles en $u$ et $v$ de forme similaire aux équations (2.5) et (2.6) se fait par l'intermédiaire d'un troisième couple d'équations en $M$ et $N$ de la façon suivante : on effectue les deux changements de variables

$$
\begin{aligned}
\eta(x, t)=\alpha^{-1} M\left(\alpha^{1 / 2} x, \alpha^{1 / 2} t\right), & \zeta(x, y, t)=\alpha^{-1} N\left(\alpha^{1 / 2} x, \alpha^{1 / 2} t\right) \\
u(x, t)=\alpha^{-1} M\left(\alpha^{-1 / 2} x+\alpha^{-3 / 2} t, \alpha^{-3 / 2} t\right), & v(x, t)=\alpha^{-1} N\left(\alpha^{-1 / 2} x+\alpha^{-3 / 2} t, \alpha^{-3 / 2} t\right) .
\end{aligned}
$$

Par contre, dans notre travail, on a remarqué qu'il suffisait, pour passer des équations de KP-II (2.1) et KPBBM-II (2.2) aux équations (2.5) et (2.6) d'effectuer le changement de fonctions

$$
u(x, y, t)=\eta\left(x+\alpha^{-1} t, y, \alpha^{-1} t\right), v(x, y, t)=\zeta\left(x+\alpha^{-1} t, y, \alpha^{-1} t\right)
$$

De plus, la donnée initiale reste toujours la même. Ce qui est important dans notre cas, c'est que l'on n'effectue aucune homothétie sur les variables d'espace $x$ et $y$, ce qui implique que nos résultats restent vrais dans le cas périodique. On obtient des résultats similaires aux propositions 2.2 et 2.3 pour le problème de Cauchy local dans le cas périodique [12]. Si on note

$M_{\pi}\left(\mathbb{R}^{2}\right):=\left\{g \in \mathcal{C}^{\infty}\left(\mathbb{R}^{2}\right), 2 \pi-\right.$ périodique en $x$ et en $y ; \int_{-\pi}^{\pi} g(x, y) \mathrm{d} x=0$ pour $\left.y \in[-\pi, \pi]\right\}$ et $X_{\pi}^{m}\left(\mathbb{R}^{2}\right)$ la fermeture de $M_{\pi}\left(\mathbb{R}^{2}\right)$ par rapport à la norme $\|g\|_{m}^{2}=\sum_{(k, l) \in \mathbb{Z}^{2}}\left(1+k^{2}+l^{2}\right)^{m}|\hat{g}(k, l)|^{2}$, on peut énoncer

Théorème 2.13. Soient $m \geq 0$, et $f \in X_{\pi}^{m+5}\left(\mathbb{R}^{2}\right)$. Soit $\alpha>0$ et soient $\eta$ et $\zeta$ l'unique solution du problème de Cauchy (2.1)-(2.2)-(2.3) dans $\mathcal{C}\left(\left[-\alpha^{-1} T_{0}, \alpha^{-1} T_{0}\right] ; X_{\pi}^{m+5}\left(\mathbb{R}^{2}\right)\right)$, avec $T_{0}:=\frac{1}{C_{m+5}|f|_{m+5}}$. Alors, il existe $\alpha_{0}>0$, et pour tous entiers naturels $0 \leq i+j \leq m$, une constante $M_{i, j}>0$, dépendant uniquement de $|f|_{m+5}$, telle que si $0<\alpha \leq \alpha_{0}$, et pour $|t| \leq \alpha^{-1} T_{0}$, on ait

$$
\left\|\partial_{x}^{i} \partial_{y}^{j} \eta(., ., t)-\partial_{x}^{i} \partial_{y}^{j} \zeta(., ., t)\right\| \leq M_{i, j} \alpha^{3 / 2}|t|^{1 / 2}
$$

Si $m \geq 2$, alors pour tous entiers naturels $0 \leq i+j \leq m-2$, il existe une constante $N_{i, j}>0$, dépendant uniquement de $|f|_{m+5}$, telle que si $0<\alpha \leq \alpha_{0}$, et pour $|t| \leq \alpha^{-1} T_{0}$, on ait

$$
\sup _{(x, y) \in[0,2 \pi]^{2}}\left|\partial_{x}^{i} \partial_{y}^{j} \eta(x, y, t)-\partial_{x}^{i} \partial_{y}^{j} \zeta(x, y, t)\right| \leq N_{i, j} \alpha^{3 / 2}|t|^{1 / 2}
$$

Remarque 2.14. Les résultats de Bona et al. [6] se généralisent à des solutions périodiques des équations de $\mathrm{KdV}$ et de BBM sur l'intervalle de temps $|t| \leq \alpha^{-1}$.

\subsection{Comparaison numérique des solutions de KP-II et KP-BBM-II}

Le but de cette partie ne sera pas d'analyser de manière approfondie le schéma numérique utilisé, mais plutôt de chercher les idées nous permettant d'établir la validité de la Conjecture 2.1. 
On considère alors le problème de Cauchy (2.5)-(2.7) avec pour donnée initiale $f$ une fonction de $X_{\pi}^{m+5}\left(\mathbb{R}^{2}\right)$, $m \geq 2$. On sait que, d'après le Théorème 2.13 , les solutions de ce problème sont telles qu'il existe $\alpha_{0}>0$, une constante $C_{m+5}$ strictement positive et dépendant uniquement de $m$, telle que si $0<\alpha \leq \alpha_{0}$, et pour $0 \leq t \leq \frac{1}{C_{m+5}|f|_{m+5}}$, on ait

$$
\|u(., ., t)-v(., ., t)\| \leq M_{0,0} \alpha t^{1 / 2} \quad \text { et } \quad \sup _{(x, y) \in[0,2 \pi]^{2}}|u(x, y, t)-v(x, y, t)| \leq N_{0,0} \alpha t^{1 / 2} .
$$

On voudrait inspecter numériquement la Conjecture 2.1, c'est-à-dire que l'on s'intéresse, d'un point de vue numérique, à l'optimalité du paramètre $0<\delta<1$ tel que, sur l'intervalle de temps $\left[0, \alpha^{-\delta}\right]$, les inégalités $(2.18)$ soient vraies. On reprend la méthode de relaxation associée à un schéma semi-implicite en temps, décrite par Besse [3], que l'on adapte aux équations de KP à l'aide d'une FFT. On fait ce choix car les méthodes de type Cranck-Nicholson ou de splitting ne sont pas judicieuses pour notre étude. En effet, Hamidouche [9] utilise une méthode de Cranck-Nicholson pour approcher les solutions des équations de KP et KP-BBM, mais pour laquelle les itérations de Picard deviennent coûteuses, d'autant plus que nous aurons besoin de faire des comparaisons pour des temps d'ordre $\alpha^{-1}$. En ce qui concerne la méthode de splitting, la résolution du problème non linéaire revient à celle de l'équation de Burgers, on risque alors de faire apparaître des chocs alors qu'il n'y en avait pas pour les équations de KP. Donc finalement, on utilise la méthode de relaxation restreinte au domaine $[-\pi, \pi] \times[-\pi, \pi]$. On réécrit les équations $(2.5)-(2.6)-(2.7)$ sous la forme

$$
\begin{aligned}
u_{t}+\frac{1}{2} \phi_{x}+u_{x x x}+\partial_{x}^{-1} u_{y y} & =0 \\
\phi & =u^{2} \\
v_{t}+\frac{1}{2} \psi_{x}+v_{x x x}-\alpha v_{x x t}+\partial_{x}^{-1} v_{y y} & =0 \\
\psi & =v^{2} \\
u(k, l, 0)=v(k, l, 0) & =f(k, l) .
\end{aligned}
$$

Soit $T_{0}$ le temps maximum de comparaison des solutions de KP-II et KP-BBM-II, et soit $\delta t$ le pas de discrétisation en temps. On note $\hat{u}^{n+1}$, respectivement $\hat{v}^{n+1}, \hat{\phi}^{n+\frac{1}{2}}, \hat{\psi}^{n+\frac{1}{2}}$, l'approximation de $\hat{u}$ au temps $(n+1) \delta t$, respectivement de $\hat{v}$ au temps $(n+1) \delta t$, de $\hat{\phi}$ au temps $\left(n+\frac{1}{2}\right) \delta t, \hat{\psi}$ au temps $\left(n+\frac{1}{2}\right) \delta t$, pour $n$ compris entre 0 et $\frac{T_{0}}{\delta t}$. En ce qui concerne les dérivées en espace, on utilise une transformée de Fourier rapide. On obtient finalement, pour $\frac{-N_{x}}{2} \leq k \leq \frac{N_{x}}{2}-1$ et $\frac{-N_{y}}{2} \leq l \leq \frac{N_{y}}{2}-1$, le schéma pour l'équation KP-II

$$
\left\{\begin{array}{l}
\frac{\hat{\phi}^{n+\frac{1}{2}}(k, l)+\hat{\phi}^{n-\frac{1}{2}}(k, l)}{2}=\widehat{\left(u^{n}\right)^{2}}(k, l) \\
\frac{\hat{u}^{n+1}(k, l)-\hat{u}^{n}(k, l)}{\delta t}+\frac{i k}{2} \hat{\phi}^{n+\frac{1}{2}}(k, l)-i\left(k^{3}-\frac{l^{2}}{k}\right) \frac{\hat{u}^{n+1}(k, l)+\hat{u}^{n}(k, l)}{2}=0 \\
\hat{u}^{n+1}(0, l)=\hat{u}^{n}(0, l)
\end{array}\right.
$$

et pour l'équation KP-BBM-II

$$
\left\{\begin{array}{l}
\frac{\hat{\psi}^{n+\frac{1}{2}}(k, l)+\hat{\psi}^{n-\frac{1}{2}}(k, l)}{2^{2}}=\widehat{\left(v^{n}\right)^{2}}(k, l) \\
\left(1+\alpha k^{2}\right) \frac{\hat{v}^{n+1}(k, l)-\hat{v}^{n}(k, l)}{\delta t}+\frac{i k}{2} \hat{\psi}^{n+\frac{1}{2}}(k, l)-i\left(k^{3}-\frac{l^{2}}{k}\right) \frac{\hat{v}^{n+1}(k, l)+\hat{v}^{n}(k, l)}{2}=0 \\
\hat{v}^{n+1}(0, l)=\hat{v}^{n}(0, l)
\end{array}\right.
$$


avec pour données initiales $\hat{u}^{0}=\hat{v}^{0}=\hat{f}$ et $\hat{\phi}^{-\frac{1}{2}}=\hat{\psi}^{-\frac{1}{2}}=\widehat{f^{2}}, N_{x}$ et $N_{y}$ sont respectivement le nombre de modes de Fourier dans les directions $x$ et $y$, et c'est aussi le nombre de points de discrétisation dans ces même directions.

L'appartenance de $f$ à $X_{\pi}^{m+5}\left(\mathbb{R}^{2}\right)$, en particulier le fait que $\int_{-\pi}^{\pi} f(x, y) \mathrm{d} x=0$, permet de pallier la singularité en $k$. On a vu lors de la modélisation que, dans les variables $u$ et $v$, les solutions des problèmes asymptotiques ne doivent pas être trop oscillantes, on choisit alors la donnée initiale de cette manière sur $[-\pi, \pi] \times[-\pi, \pi]$ et de telle sorte que sa norme $L^{2}\left(\mathbb{R}^{2}\right)$ soit égale à 1 , les pas de discrétisation en espace n'ont alors pas besoin d'être trop élevés. On aura remarqué lors de nos tests que pour les nombres de points de discrétisation en espace $N_{x}$ et $N_{y}$, pris égaux ou non parmi 64 et 128, les résultats sont sensiblement les mêmes. On remarque la même chose pour un pas de discrétisation en temps $\delta t$ pris aux alentours de $10^{-3}$ et 0.05 . On choisit d'illustrer les résultats pour $N_{x}=128, N_{y}=128$ et $\delta t=0.05$. Le temps maximum de comparaison théorique est de l'ordre d'une constante, on effectue alors des itérations pour un intervalle de temps d'ordre $\alpha^{-1}$. Dans un premier temps, on observera la comparaison numérique du problème non linéaire, ainsi que du problème linéaire. On espère alors que le temps de comparaison du problème non linéaire soit meilleur que le temps théorique mais devrait être moins bon que le temps de comparaison du problème linéaire. Dans un second temps, on effectue la comparaison du problème non linéaire pour différents choix de $\alpha$. Le but sera de voir si le temps de comparaison dépend ou non de $\alpha$.

On effectue les tests (voir Fig. 1) pour

$$
f(x, y)=\frac{\cos (x+y)}{\|\cos (x+y)\|}, \quad N x=128, \quad N y=128, \quad \delta t=0.05, \quad \alpha=10^{-3} .
$$

On remarque une très forte croissance des normes à peu près à partir du temps $t=140$, c'est-à-dire pour $\delta=\frac{\ln 14}{3 \ln 10}+\frac{1}{3} \simeq 0,715$. On effectue un second test (voir Fig. 1) pour

$$
f(x, y)=\frac{\cos x \cos y}{\|\cos x \cos y\|}, \quad N x=128, \quad N y=128, \quad \delta t=0.05, \quad \alpha=10^{-3} .
$$

On remarque pour ce dernier test une très forte croissance des normes à peu près à partir du temps $t=260$, soit $\delta=\frac{\ln 26}{3 \ln 10}+\frac{1}{3} \simeq 0.804$, ce qui reste bien meilleur que le temps théorique. En ce qui concerne la comparaison numérique du problème linéaire, on observe dans tous nos tests que les inégalités (2.18) restent vraies pour toutes les itérations, ceci est du au caractère périodique des solutions du problème linéaire.

Reprenons les tests précédents en augmentant cette fois-ci le nombre d'oscillations de la donnée initiale, résumons tout cela dans un tableau (Tab. 1).

Effectuons la deuxième série de tests. Il s'agira de voir l'influence du paramètre $\alpha$ et aussi de trouver une borne pour la valeur limite $\alpha_{0}$. Choisir la valeur $\alpha_{\star}=2.5 \times 10^{-4}$ correspond sensiblement à ce qui est observé dans l'océan pacifique, à savoir des ondes de surface d'une amplitude de 1 mètre, de longueur d'onde 213 kilomètres dans la direction $x$, pour une profondeur de 4000 mètres [10]. On compare ces choix aux valeurs $\alpha=10^{-2}, 10^{-3}$ et $10^{-4}$ dans nos tests (voir Fig. 3). On se limite à des valeurs de $\alpha$ supérieures à $10^{-4}$, car lorsque $\alpha$ est très petit, on perd la notion de petitesse de $\alpha$ au profit d'erreurs de calcul du programme. Il serait intéressant de trouver un schéma numérique mieux adapté aux calculs pour $\alpha$ très petit.

La valeur $10^{-2}$ apparaît comme une borne dans le choix de $\alpha_{0}$ vérifiant, pour $0<\alpha \leq \alpha_{0}$, qu'il existe $0<\delta<1$ tel que les inégalités (2.18) soient vraies sur l'intervalle de temps $0<t<\alpha^{-\delta}$. En effet, on note que, dans tous les cas, le temps de comparaison ne change pas selon la valeur de $\alpha$, mais ce temps étant donné par la relation $t=\alpha^{-\delta}$, on trouve :

- avec la donnée initiale $f(x, y)=\frac{\cos (x+y)}{\|\cos (x+y)\|}, \delta$ est égal à 1.073, 0.715, 0.596 et 0.544 ;

- avec la donnée initiale $f(x, y)=\frac{\cos x \cos y}{\|\cos x \cos y\|}, \delta$ est égal à $1.207,0.804,0.670$, et 0.604 , 

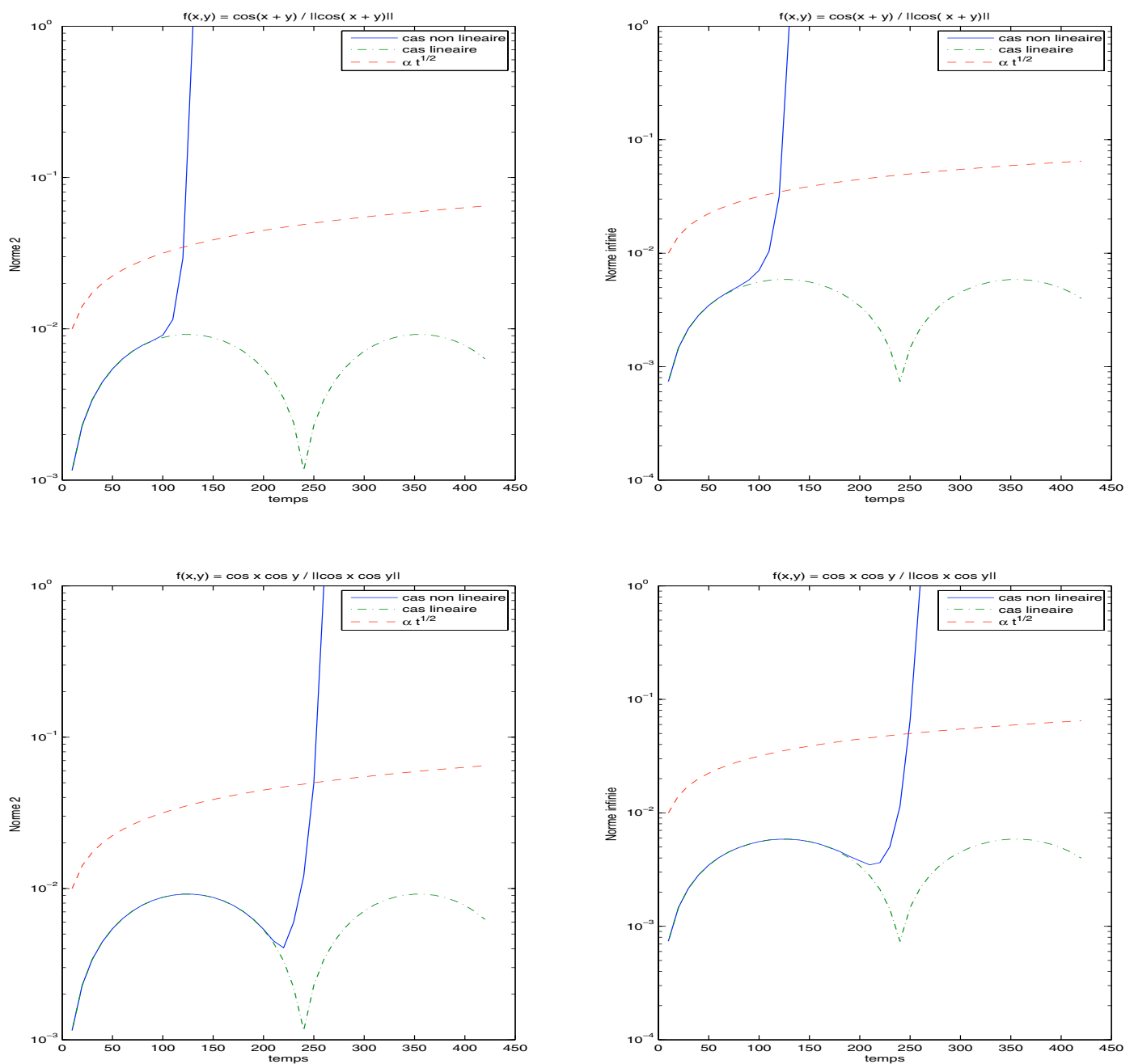

Figure 1. Comparaison numérique des normes $L^{2}$ et $L^{\infty}$ pour les problèmes linéaire et non linéaire avec $\alpha=10^{-3}$ sur l'intervalle de temps $\left[0, \alpha^{-1}\right]$ et avec les données initiales $f(x, y)=\frac{\cos (x+y)}{\|\cos (x+y)\|}$ et $f(x, y)=\frac{\cos x \cos y}{\|\cos x \cos y\|}$ en utilisant l'échelle logarithmique sur $O y$.

respectivement pour $\alpha$ égal à $10^{-2}, 10^{-3}, 2.5 \times 10^{-4}$, et $10^{-4}$. Essayons de voir plus précisément quel serait le cas limite dans le choix de $\alpha_{0}$. Pour cela, on reprend les tests pour différentes valeurs de $\alpha$ proche de $10^{-2}$.

Il résulte des simulations effectuées que la borne $\alpha_{0}$ est proche de $7.14 \times 10^{-3}$ et $3.84 \times 10^{-3}$, respectivement pour les données initiales $f(x, y)=\frac{\cos (x+y)}{\|\cos (x+y)\|}$ et $f(x, y)=\frac{\cos x \cos y}{\|\cos x \cos y\|}$ (voir Fig. 2). On a vu aussi que ces bornes numériques n'engendrent pas de limitation dans les choix physiques possibles de valeurs de $\alpha$, étant donné que ces deux cas ne sont pas réalistes.

Pour les données initiales $\frac{\sin (x+y)}{\|\sin (x+y)\|}$ et $\frac{\sin x \sin y}{\|\sin x \sin y\|}$, on obtient des résultats similaires. 
Tableau 1. Temps maximum de comparaison en fonction du nombre d'oscillations de la donnée initiale.

\begin{tabular}{|c|c|c||c|c|c|}
\hline $\begin{array}{c}\text { Donnée initiale } \\
f(x, y)\end{array}$ & $\begin{array}{c}\text { Temps maximum } \\
\text { de comparaison } \\
\text { non linéaire }\end{array}$ & Paramètre $\delta$ & $\begin{array}{c}\text { Donnée initiale } \\
f(x, y)\end{array}$ & $\begin{array}{c}\text { Temps maximum } \\
\text { de comparaison } \\
\text { non linéaire }\end{array}$ & Paramètre $\delta$ \\
\hline \hline$\frac{\cos (x+y)}{\|\cos (x+y)\|}$ & 140 & $\delta \simeq 0.715$ & $\frac{\cos x \cos y}{\|\cos x \cos y\|}$ & 260 & $\delta \simeq 0.804$ \\
\hline$\frac{\cos (2 x+y)}{\|\cos (2 x+y)\|}$ & 130 & $\delta \simeq 0.704$ & $\frac{\cos 2 x \cos y}{\|\cos 2 x \cos y\|}$ & 260 & $\delta \simeq 0.804$ \\
\hline$\frac{\cos (x+2 y)}{\|\cos (x+2 y)\|}$ & 140 & $\delta \simeq 0.715$ & $\frac{\cos x \cos 2 y}{\|\cos x \cos 2 y\|}$ & 260 & $\delta \simeq 0.804$ \\
\hline$\frac{\cos (2 x+2 y)}{\|\cos (2 x+2 y)\|}$ & 130 & $\delta \simeq 0.704$ & $\frac{\cos 2 x \cos 2 y}{\|\cos 2 x \cos 2 y\|}$ & 250 & $\delta \simeq 0.799$ \\
\hline
\end{tabular}
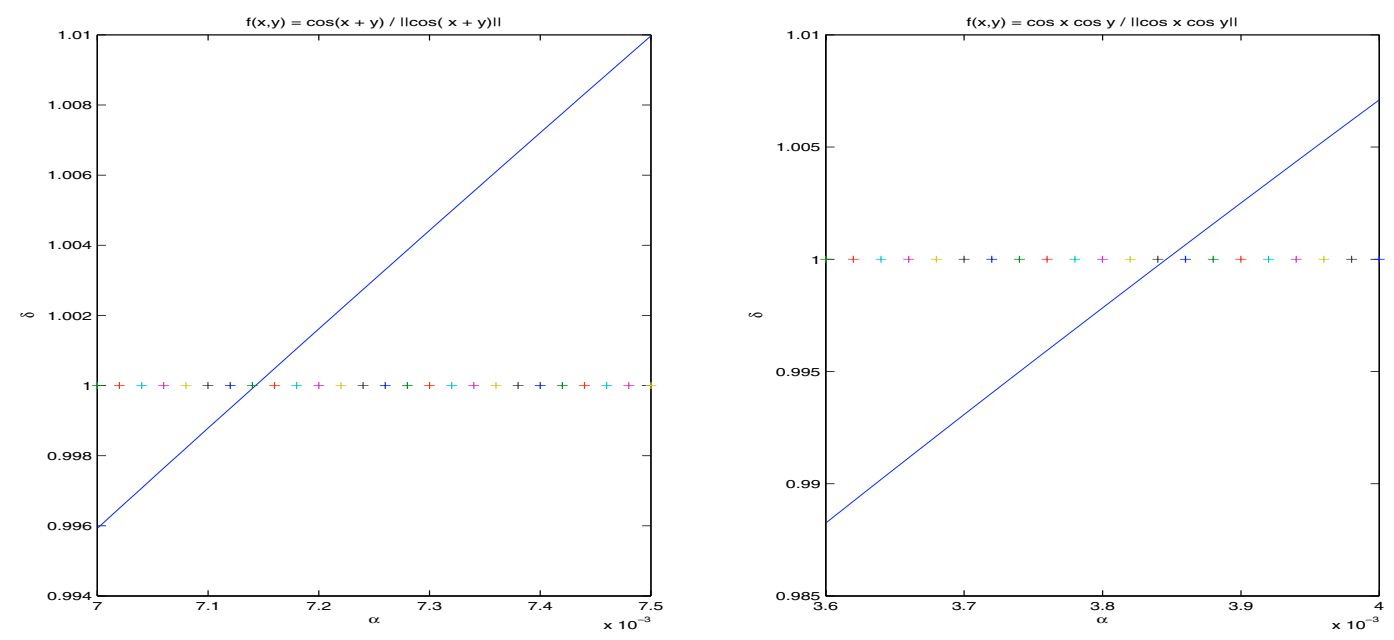

Figure 2. Evolution de $\delta$ en fonction de $\alpha$ pour le problème non linéaire avec les données initiales $f(x, y)=\frac{\cos (x+y)}{\|\cos (x+y)\|}$ et $f(x, y)=\frac{\cos x \cos y}{\|\cos x \cos y\|}$.

Il semblerait que la Conjecture 2.1 soit vraie, il reste alors à montrer jusqu'où on peut prolonger le temps de comparaison des solutions de KP-II et KP-BBM-II, on peut tout de même faire quelques observations : 

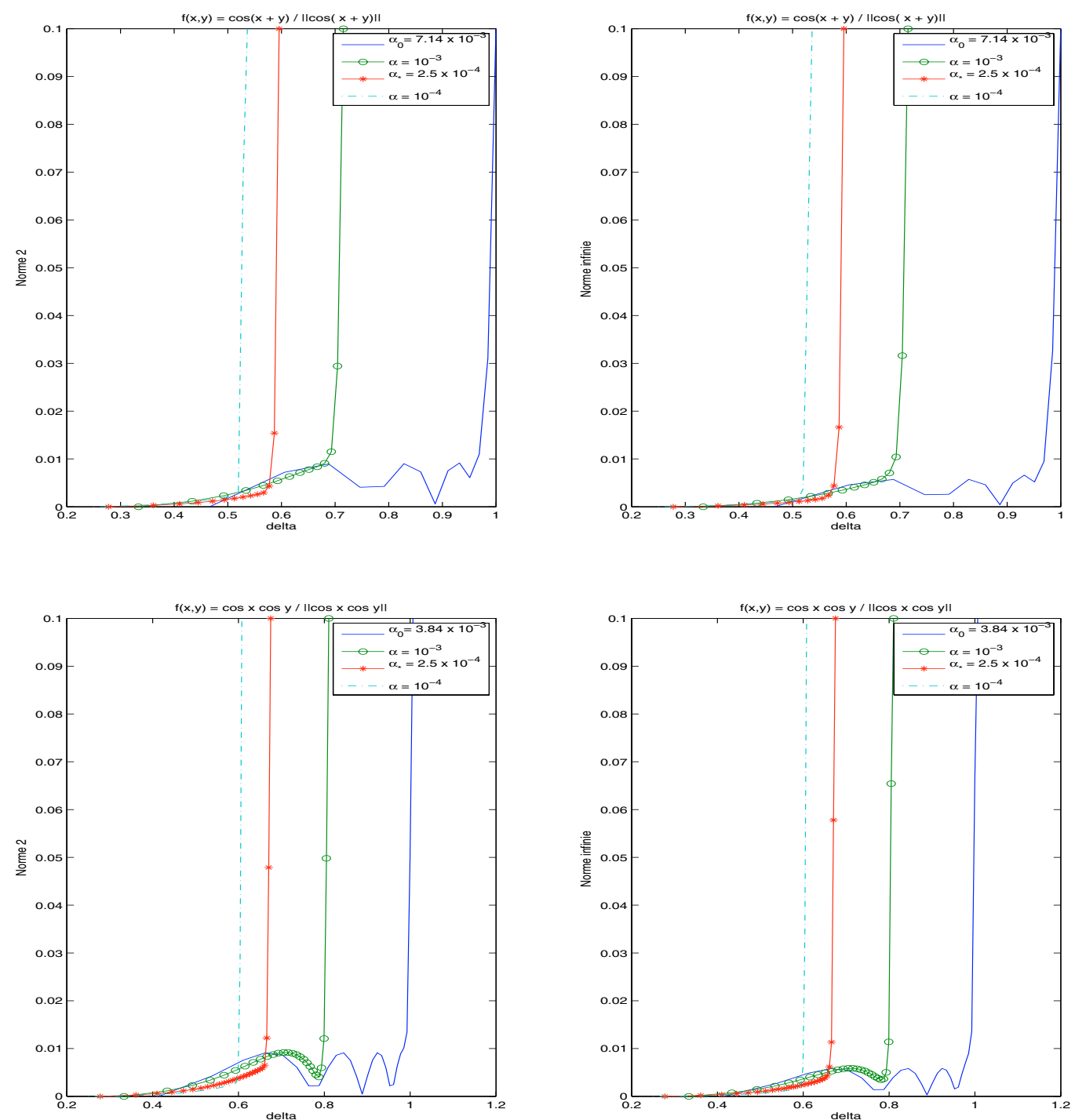

Figure 3. Comparaison numérique des normes $L^{2}$ et $L^{\infty}$ pour le problème non linéaire en fonction de $\delta$ avec $\alpha=10^{-3}, 2.5 \times 10^{-4}, 10^{-4}$, et $\alpha_{0}=7.14 \times 10^{-3}, 3.84 \times 10^{-3}$ respectivement pour chacune des données initiales $f(x, y)=\frac{\cos (x+y)}{\|\cos (x+y)\|}$ et $f(x, y)=\frac{\cos x \cos y}{\|\cos x \cos y\|}$.

- le nombre d'oscillations de la donnée initiale dans les directions $x$ et $y$, qui ne doit pas être trop élevé de part la modélisation, n'influence pas la comparaison;

- la comparaison du problème non linéaire, lors des premiers instants d'observation, a un comportement sensiblement proche de celle du problème linéaire;

- le paramètre $\delta$ est plus ou moins proche de 1 que de 0 , autrement dit le temps de comparaison est plus proche du temps donné par le problème linéaire que du temps théorique, selon que $\alpha$ est plus ou moins proche de $\alpha_{0}$. De plus, cette valeur limite $\alpha_{0}$ semble dépendre de la donnée initiale; 
- si $\alpha$ était nul, les équations de KP-II et KP-BBM-II se réduisant à la seule équation des ondes, les solutions respectives seraient donc les mêmes et $\delta$ vaudrait 1 ;

- finalement, on constate que pour des données semblables à ce que l'on observe dans la réalité, c'est-à-dire pour $10^{-4} \leq \alpha \leq 10^{-3}$, les solutions des équations de KP-II et KP-BBM-II sont suffisamment proches l'une de l'autre sur un intervalle plus grand que le temps théorique pour cette modélisation du problème physique.

Remerciements. L'auteur tient à remercier le Professeur Nikolay Tzvetkov pour son aide et sa grande disponibilité, ainsi que le Professeur Christophe Besse. Un remerciement tout particulier à Seraphin Mefire pour son accueil et nos discussions. Merci aussi à Pauline Lafitte-Godillon pour ses remarques.

\section{RÉFÉRENCES}

[1] J. Ablowitz and H. Segur, On the evolution of packets of water waves. J. Fluid Mech. 92 (1979) 691-715.

[2] T.B. Benjamin, J.L. Bona and J.J. Mahony, Model equations for long waves in nonlinear dispersive systems. Phil. Trans. R. Soc. London Ser. A 272 (1972) 47-78.

[3] C. Besse, A relaxation scheme for the nonlinear Shrödinger equation. J. Numer. Anal. 42 (2004) 934-952.

[4] J.L. Bona and H. Chen, Comparison of model equations for small-amplitude long waves. Nonlinear Anal. 38 (1999) 625-647.

[5] J.L. Bona and R. Smith, The initial-value problem for the Korteweg-de Vries equation. Philos. Trans. Roy. Soc. London Ser. A 278 (1975) 555-601.

[6] J.L. Bona, W.G. Pritchard and L.R. Scott, A comparison of solutions of two model equations for long waves. Lect. Appl. Math. 20 (1983) 235-267.

[7] J.L. Bona, Y. Liu and M.M. Tom, The Cauchy problem and stability of solitary wave solutions for the RLW-KP equation. $J$. Differ. Equations 185 (2002) 437-482.

[8] J. Bourgain, On the Cauchy problem for the Kadomtsev-Petviashvili equation. Geom. Funct. Anal. 3 (1993) 315-341.

[9] F. Hamidouche, Simulations numériques des équations de Kadomtsev-Petviashvili. Thèse de doctorat, Université Paris XI, France (2001).

[10] International Tsunami Information Centre, Tsunami : les grandes vagues. Brochure du programme Tsunami de l'UNESCO (2003).

[11] R.J Iório Jr., KdV, BO and friends in weighted Sobolev spaces. Lect. Notes Math. 1450 (1990) 104-121.

[12] R.J Iório Jr. and W.V.L. Nunes, On equation of KP-type. Proc. Roy. Soc. Edinburgh A 128 (1998) 725-743.

[13] B.B. Kadomtsev and V.I. Petviashvili, Model equations for long waves in nonlinear dispersive systems. Sov. Phys. Dokady 15 (1970) 539

[14] T. Kato and G. Ponce, Commutator estimates for the Euler and Navier-Stokes Equations. Comm. Pures. Appl. Math. 14 (1988) 891-907.

[15] D.J. Korteweg and G. de Vries, On the change of form of long waves advancing in a rectangular canal and on a new type of long stationnary waves. Phil. Maj. 39 (1895) 422-443.

[16] M.D. Kruskal, Nonlinear wave equations, theory and applications. Lect. Notes Phys. (1975) 310-354.

[17] A. Miranville, R. Temam, Mathematical Modelling in Continuum Mechanics. Cambridge University Press (1999).

[18] J.C. Saut and N. Tzvetkov, Global well-posedness for the KP-BBM equation. AMRX (2004) 1-16.

[19] M.E. Taylor, Partial Differential Equations I : Basic theory. Applied mathematical sciences 115, Springer (1996).

[20] G.B. Whitham, Linear and Nonlinear Waves. John Wiley \& Sons, New York (1974). 